\title{
Charakterisierung eines Gebiets Durch SpekTRAldaten EINES Dirichletproblems ZUR STOKESGLEICHUNG
}

\author{
Dissertation \\ zur Erlangung des Doktorgrades \\ der Mathematisch-Naturwissenschaftlichen Fakultäten \\ der Georg-August-Universität zu Göttingen \\ vorgelegt von \\ Viktor Tsiporin \\ aus \\ St. Petersburg, Russland
}

Göttingen 2003 
D7

Referent: Prof. Dr. Rainer Kress

Korreferent: PD Dr. Roland Potthast

Tag der mündlichen Prüfung: 20.01.2004 


\section{Inhaltsverzeichnis}

$\begin{array}{lll}1 & \text { Einführung } & 2\end{array}$

\begin{tabular}{lll}
\hline 2 & Direktes Problem & 7
\end{tabular}

2.1 Grundlagen zur Stokes Gleichung . . . . . . . . . . . . . . . 7

$2.1 .1 \quad$ Die Fundamentallösung und der Greensche Satz . . . . . . . . 7

2.1.2 $\quad$ Die hydrodynamischen Potentiale $\ldots \ldots \ldots \ldots$

2.2 Äusseres Dirichlet Problem bei $C^{1}$-Randdaten . . . . . . . . . . . . . . 14

2.3 Äusseres Dirichlet Problem bei $L^{2}$-Randdaten . . . . . . . . . . . . . 19

$2.4 \quad$ Numerische Behandlung des ÄDP im $\mathbb{R}^{2} \ldots \ldots \ldots$. . . . . . . 23

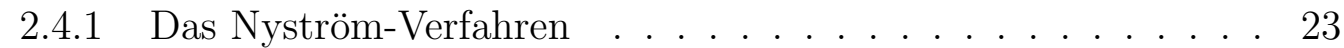

2.4 .2 Numerische Beispiele $\ldots \ldots \ldots$. . . . . . . . . . . 26

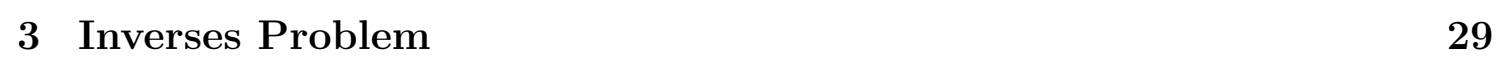

3.1 Der dreidimensionale Fall . . . . . . . . . . . . . . . . . . . . . . 30

$3.1 .1 \quad$ Faktorisierung des Meßoperators $\ldots \ldots \ldots \ldots$

3.1 .2 Charakterisierung des Gebiets $\ldots \ldots \ldots \ldots$. . . . . . . 34

3.2 Der zweidimensionale Fall $\ldots \ldots \ldots \ldots . \ldots \ldots$

$3.2 .1 \quad$ Faktorisierung des Meßoperators . . . . . . . . . . . 37

3.2 .2 Charakterisierung des Gebiets . . . . . . . . . . . . . . 41

\begin{tabular}{|lll}
\hline & Numerische Umsetzung & 46
\end{tabular}

4.1 Rekonstruktion durch Reihenwertvergleich . . . . . . . . . . . . . 47

4.2 Das exponentielle Abklingen der Eigenwerte $\ldots \ldots \ldots \ldots$

$\begin{array}{ll}\text { Literaturverzeichnis } & 53\end{array}$ 


\section{Kapitel 1}

\section{Einführung}

Probleme zu lösen, die eine Beschreibung der Reaktion von physikalischen Systemen auf eine Wirkung von Aussen erfordern, war lange Zeit die vorrangige Aufgabe der mathematischen Physik. Der Umfang des auf diesem Gebiet angesammelten Wissens war zu Beginn der 1970er Jahre ausreichend, damit die Wissenschaft mit der Untersuchung einer neuen Klasse von Problemen anfangen konnte: Aus der Kenntnis der Reaktion eines Systems auf dessen Parameter zu schliessen. Die Fragestellungen dieser Art nennt man inverse Probleme, wodurch hervorgehoben wird, daß deren Formulierungen die Kenntnis der Lösung des entsprechenden direkten, i.d.R. einfacheren bzw. bereits untersuchten Problems voraussetzt.

Ein solches inverses Problem wird auch in der vorliegenden Arbeit behandelt: Das mit dem äußeren Dirichlet Problem zur Stokes-Gleichung (dem direkten Problem) verbundene inverse Streuproblem erfordert die Rekonstruktion der Form eines unbekannten Objekts aus den Messungen des Ansprechverhaltens der Geschwindigkeitsfelder bei auf einer das Objekt umliegenden Kurve gesetzten Quellen und Meßstellen. Dieses Problem fällt in die Klasse der inversen Streuprobleme.

Die Stokes-Gleichung für das Geschwindigkeit-Druck-Paar $(u, p)$

$$
\left\{\begin{array}{c}
-\Delta u+\operatorname{grad} p=0 \\
\operatorname{div} u=0
\end{array}\right.
$$

gibt eine adäquate Beschreibung von Strömungen inkompressibler Newtonscher Flüssigkeiten bei sehr niedrigen Reynoldszahlen, die wegen der typisch äußerst kleinen Geschwindigkeiten allgemein Kriechströmungen genannt werden. Sie bildet das Hauptmodell zur Beschreibung der Bewegung von Mikroorganismen (s.g. microswimmers), der geophysikalischen Strömungen oder aber auch der Sedimentation von Partikeln. Zu der linearen Stokes-Gleichung 1.1 führt, aus mathematischer Sicht, die Vernachlässigung des konvektiven Terms in den allgemeinen Navier-Stokes-Gleichungen 
und die Annahme, daß die Flüssigkeitsdichte bei einem stationären Fluß verschwin$\operatorname{det}($ s. z. B. $[\mathrm{POZ}])$.

Das direkte Problem ist in der Literatur recht gut untersucht. Wie bei vielen Randwertaufgaben der mathematischen Physik bilden die Integralgleichungsmethoden auch hierbei ein bedeutendes Hilfsmittel. Eine state-of-the-art-Behandlung der mit 1.1 verbundenen Randwertprobleme mit der Randintegralgleichungsmethode findet man in Power/Wrobel.

Unser Zugang zu dem inversen Problem basiert auf der Faktorisierungsmethode, die 1998 von A. Kirsch als Modifikation der linear sampling Methode vorgeschlagen wurde, um unterschiedliche Typen von inversen Streuproblemen bei der Helmholtz Gleichung zu behandeln (siehe z.B. Kirsch1]). Während die meisten klassischen Algorithmen zur Gebietsrekonstruktion entweder einen iterativen Charakter haben (die Methoden Newtonschen oder Landweberschen Typs) und wiederholtes Lösen der direkten Probleme erfordern, oder den sogenannten Problemdekompositionszugang benutzen (dual-space-method, die Technik von Kirsch-Kreß, die Punktquellenmethode), gehört die Faktorisierungsmethode zu einer relativ neuen Klasse von bildgebenden Singularitätsmethoden, deren Ziel die punktweise Rekonstruktion des zu visualisierenden Streuobjekts ist. Für weitere Methoden dieser Klasse sei hier stellvertretend auf die Publikationen [Ikehata'98], [Potthast'01], [Luke/Potthast] verwiesen.

Bezogen auf den dreidimensionalen Fall des von uns behandelten inversen Problems läßt sich die Faktorisierungsmethode wie folgt beschreiben:

Für ein beschränktes Gebiet $D$ in $\mathbb{R}^{3}$ mit zusammenhängendem $C^{3}$-Rand $\partial D$ und ein $D$ enthaltendes $C^{2}$-glattes beschränktes Gebiet $B$ sind die Lösungen $u(x)$ der äußeren Dirichlet Probleme zu 1.1 mit den Randdaten

$$
u_{i}=-\Psi_{3}^{(r, c)}(\cdot-z) \cdot e_{i} \text { auf } \partial D
$$

für alle $x, z \in \partial B$ bekannt, wobei mit $e_{i}, i=1, \ldots, 3$ die Einheitsvektoren in $\mathbb{R}^{3}$ bezeichnet sind und $\Psi_{3}^{(r, c)}$ die Fundamentallösung der Stokesgleichung ist (s. Kapitel 2). Faßt man diese Lösungen zu einer Antwortsmatrix w $(x, y)$ zusammen, so zeigt die Untersuchung des Meßoperators $F_{3}:\left(L^{2}(\partial B)\right)^{3} \rightarrow\left(L^{2}(\partial B)\right)^{3}$, definiert als

$$
\left(F_{3} g\right)(x):=-\int_{\partial B} w(x, y) g(y) d s(y) \quad x \in \partial B,
$$

daß er kompakt, selbstadjungiert und positiv semi-definit ist und somit eine eindeutig definierte Quadratwurzel $F_{3}^{\frac{1}{2}}$ besitzt. Ordnet man jedem Punkt $z \in B$ die Funktion $f_{z}(x):=\Psi^{(r, c)}(x-z) a, \quad x \in \partial B$ mit einem $a=$ const $\in \mathbb{R}^{3}, a \neq 0 \mathrm{zu}$, so läßt sich beweisen, daß $z$ dann und nur dann in $D$ liegt, wenn die Gleichung

$$
F_{3}^{\frac{1}{2}} g=f_{z}
$$


eine Lösung in $\left(L^{2}(\partial B)\right)^{3}$ besitzt. Die Entscheidung, ob die Lösung von 1.2 in $\left(L^{2}(\partial B)\right)^{3}$ liegt, ist der Konvergenzuntersuchung für ihre Fourier-Reihe (der Entscheidungsreihe) nach der hergeleiteten Basis gleichzusetzen.

Der Hauptvorteil der Methode von Kirsch gegenüber den iterativen Techniken besteht im Allgemeinen darin, daß keine a priori Information über die Form des Objekts und die Zahl seiner Komponenten benötigt wird, und nicht einmal die Kenntnis des Typs der Randbedingung erforderlich ist. Dabei zeichnet sich die Faktorisierungsmethode durch eine hohe numerische Effizienz aus: die Behandlung eines nichtlinearen Problems wird anhand der Eigenschaften eines linearen Operators vorgenommen, wobei kein numerischer Linearisierungsvorgang stattfindet.

Nicht erstaunlich ist also, daß die Anwendbarkeit dieser Methode und ihrer Varianten bei verschiedenen physikalischen Gegebenheiten derzeit aktiv überprüft wird, vor allem im Bereich der inversen Streuprobleme. Uns sind bereits folgende Resultate in diesem Bezug bekannt:

- für die skalare zweidimensionale Helmholzgleichung bei Fernfelddaten im ganzen Raum ([Kirsch3]) und im geschichteten Medium ( Coyle]), bei Nahfelddaten für Freiraum und Halbraum ([Kirsch3], [CCM] ) sowie für unbeschränkte periodische Streuobjekte ([Arens/Kirsch $)$;

- für einige Vektorfeldformulierungen [CHM], HMO] (elektromagnetische Wellen, Maxwellgleichungen), Kress3] (harmonische Vektorfelder);

- bei der Laplace-Gleichung für das inverse Dirichlet Problem in $\mathbb{R}^{3}$ ([Hähner1]) und in $\mathbb{R}^{2}$ ([Kress1], [Kress2]), für das inverse Neumann Problem in $\mathbb{R}^{2}([\mathrm{KK}])$ sowie für das impedanztomographische Problem ([Brühl], [BRH]).

- Eine Erweiterung der Faktorisierungsmethode auf inverse Streuprobleme für elastische Wellen wurde von Alves und Kress ([AK]) sowie von Arens ([Arens]) durchgeführt.

In diese (bestimmt nicht vollständige) Liste fügt sich auch die vorliegende Arbeit. Wir geben eine theoretische Begründung der Anwendbarkeit der Faktorisierungsmethode auf den Fall der Stokes Gleichung und illustrieren sie anhand numerischer Beispiele. Die Motivation zu dieser Untersuchung beinhaltet zwei Aspekte: $1^{\circ}$ Das direkte Problem ist vektoriell, $2^{o}$ die Faktorisierungsmethode wurde bisher auf kein inverses Streuproblem der Hydrodynamik angewendet.

Im Kapitel 2 sind, vor allem aus vorhandener Literatur zusammengestellt, Ergebnisse der klassischen Lösbarkeitstheorie für die mit der Stokesgleichung 1.1 verbundenen Randwertprobleme vorgestellt, soweit diese für die Behandlung unseres inversen Problems relevant sind. Zusätzlich werden zwei neue Aussagen bewiesen. 
Zunächst wird ein Überblick über den potentialtheoretischen Zugang zur Behandlung des Äusseren Dirichlet Problems ( ̈̈DP) gegeben, wobei als erstes die grundlegenden Fragen von Existenz und Eindeutigkeit klassischer Lösungen beantwortet werden. Im Hinblick auf das inverse Problem ist uns vor allem der zum Existenznachweis benutzte Apparat von Interesse. Als nächstes überzeugen wir uns, daß dieser auch bei quadratsummierbaren Randdaten verwendbar bleibt. Dabei beweisen wir ein Resultat über die Eindeutigkeit der Lösung des Äußeren Dirichlet Problems bei $L^{2}$-Randdaten und untersuchen die Sprungbeziehungen für die Stokes-Potentiale bei Dichten in $L^{2}$.

Am Ende des Kapitels wird das numerisches Lösungsverfahren (das Nyström Verfahren) für das ÄDP beschrieben, das uns die synthetischen Ausgangsdaten für das inverse Problem liefert. Durch mehrere Beispiele demonstrieren wir die Effizienz dieses Verfahrens beim numerischen Lösen des Vorwärtsstreuproblems.

Das Kapitel 3 ist der theoretischen Begründung der Faktorisierungsmethode für das inverse Problem gewidmet. Nach der mathematischen Formulierung des Problems wird als erstes der dreidimensionale Fall untersucht. Dies erfolgt in zwei Schritten:

$1^{o}$ Für den Meßoperator wird eine faktorisierte Form $F_{3}=G S G^{*}$ mit einem Hilfsoperator $G$ und den direkten Werten des Einfachschichtpotentials $S$ hergeleitet, aus welcher seine Abblidungseigenschaften durch Untersuchung der Faktoren ersichtlich werden. Auf diesem Wege wird u.a. die positive Definitheit von $F_{3}$ gezeigt und sein Nullraum beschrieben.

$2^{o}$ Anhand der festgestellten Eigenschaften des Meßoperators und seiner Eigenfunktionen wird eine vollständige orthonormale Basis des Raumes

$$
L_{\perp \nu}^{2}(\partial B):=\left\{\varphi \in\left(L^{2}(\partial B)\right)^{3}:<\varphi,\left.\nu\right|_{\partial B}>_{L^{2}(\partial B)}=0\right\}
$$

konstruiert (hier steht $\left.\nu\right|_{\partial B}$ für die äußere Einheitsnormale zu $\partial B$ ).

Mit dieser Basis beweisen wir die Hauptaussage des Abschnitts 3.1 - ein Kriterium für die Zugehörigkeit eines Punktes zum gesuchten Objekt im Sinne der Lösbarkeit der Gleichung 1.2 in $L_{\perp \nu}^{2}(\partial B)$.

Der etwas kompliziertere zweidimensionale Fall ist im Abschnitt 3.2 behandelt. Wegen des logarithmischen Verhaltens der Grundlösung im Unendlichen muß der Einfachschichtpotentialansatz zur Darstellung beschränkter Lösungen des Vorwärtsproblems modifiziert werden (vgl. Bemerkung 3.1). Die zur Untersuchung des zweidimensionalen Meßoperators (s. Teilabschnitt 3.2.1) notwendige Faktorisierung verändert sich entsprechend. Nach deren Herleitung konnten wir zeigen, daß auch der zweidimensionale Meßoperator kompakt, selbstadjungiert und positiv semi-definit mit dreidimensionalem Nullraum ist. 
Nach dem Nachweis der Lösbarkeit des beschränkten äußeren Dirichlet Problems mit Hilfe des abgeänderten Einfachschichtpotentialansatzes ist im Teilabschnitt 3.2.2 das dem dreidimensionalen Fall analoge Entscheidungskriterium bewiesen.

Im Kapitel 4 ist die numerische Umsetzung des inversen Verfahrens beschrieben. Der sich aus der Theorie ergebende Algorithmus wird mit zwei verschiedenen Ansätzen implementiert. Der Vorstellung der numerisch erzielten Ergebnisse bei der eher intuitiven Rekonstruktion durch Reihenwertvergleich folgen Beispiele, welche das erwartete exponentielle Abklingen der Eigenwerte des Meßoperators zur Konvergenzuntersuchung der Entscheidungsreihe benutzen. 


\section{Kapitel 2}

\section{Direktes Problem}

\subsection{Grundlagen zur Stokes Gleichung}

\subsubsection{Die Fundamentallösung und der Greensche Satz}

Der Fundamentaltensor $\Psi=\left(\Psi_{j k}\right)_{j, k=1, \ldots, m+1}$ des Stokes-Systems 1.1 im $\mathbb{R}^{m}, m \geq 2$, das sogenannte Stokeslet!1 lautet

$$
\begin{aligned}
& \Psi_{j k}=\frac{1}{2 \omega_{m}}\left\{\frac{x_{j} x_{k}}{|x|^{m}}+\delta_{j k}\left\{\begin{array}{cc}
\ln \frac{1}{|x|} & (m=2) \\
\frac{|x|^{2-m}}{m-2} & (m \geq 3)
\end{array}\right\}\right\}, j, k=1, \ldots, m \\
& \Psi_{m+1, k}=\Psi_{k, m+1}=\frac{x_{k}}{\omega_{m}|x|^{m}}, k=1, \ldots, m, \quad \Psi_{m+1, m+1}=\delta(x)
\end{aligned}
$$

mit der Diracschen Distribution $\delta$ und der Oberfläche $\mathbb{R}^{m} \omega_{m}=2 \pi^{\frac{m}{2}} / \Gamma\left(\frac{m}{2}\right)$ der $(m-1)$-dimensionalen Sphäre im $\mathbb{R}^{m}$. Für die Herleitung des Fundamentaltensors s. z.B. [Lad].

Mit $\Psi^{(r, c)}$ wird in dieser Arbeit die führende Hauptmatrix (die Geschwindigkeitskomponente) von $\Psi$ bezeichnet. Das Symbol $r$ bedeutet hier „ohne letzte Zeile“, $c$ - „ohne letzte Spalte“. Desweiteren verwenden wir folgende Notationen: für ein Funktionenpaar $(u, p), \mathrm{u}$ - vektorwertig, $\mathrm{p}$ - skalar, seien

$$
\mathcal{S}:\left(\begin{array}{l}
u \\
p
\end{array}\right) \rightarrow \mathcal{S}_{p}^{u}:=\left(\begin{array}{c}
-\Delta u+\nabla p \\
\nabla \cdot u
\end{array}\right)
$$

der formelle Stokes-Operator,

\footnotetext{
${ }^{1}$ Physikalisch entspricht das Stokeslet dem von einer Punktquelle erzeugtem Fluss.
} 


$$
D u:=\frac{1}{2}\left(\nabla u+\nabla^{T} u\right)
$$

der Deformationstensor und

$$
T:\left(\begin{array}{l}
u \\
p
\end{array}\right) \rightarrow T_{p}^{u}:=-p I_{m}+2 D u
$$

der dem Fluss $(u, p)$ entsprechende Spannungstensor.

Mit diesen Bezeichnungen lässt sich das hydrodynamische Analogon des ersten Greenschen Satzes wie folgt formulieren:

\section{Satz 2.1}

Für ausreichend glatte, divergenzfreie Vektorfelder $u, v$ und skalare Funktionen $p, q$ in einem beschränkten Gebiet $D \in \mathbb{R}^{m}$ mit $\partial D \in C^{1}$ gilt (s. [Lad])

$$
\int_{D}\left(\mathcal{S}_{p}^{u}\right) \cdot\left(\begin{array}{l}
v \\
q
\end{array}\right) d y=-\int_{\partial D}\left(T_{p}^{u} \nu\right) \cdot v d s(y)+2 \int_{D} D u: D v d y .
$$

Dabei steht $\nu$ für die äussere (bzgl. $D$ ) Einheitsnormale zu $\partial D$ und die Operation ":" für zwei $n \times n$-Matrizen $A=\left(A_{i j}\right), B=\left(B_{i j}\right)$ ist erklärt als

$$
A: B=\sum_{i, j=1}^{n} A_{i j} B_{i j}
$$

Ist $(u, p)$ ein Stokes-Fluss, d.h. es gilt $\mathcal{S}_{p}^{u}=0$ in $D$, so folgt aus $(2.3)$ sofort die nützliche Beziehung

$$
\int_{\partial D}\left(T_{p}^{u} \nu\right) \cdot u d s(y)=2 \int_{D} D u: D u d y,
$$

welche das Energieerhaltungsgesetz zum Ausdruck bringt: die rechte Seite dieser Gleichung entspricht dem Verlust der mechanischen Energie im Kontrolvolumen $D$ und die linke Seite beschreibt die Arbeit der Oberflächenkraft auf $D$ (s. [POZ]).

\subsubsection{Die hydrodynamischen Potentiale}

Die Singularitäten in der Grundlösung der Stokes-Gleichung und in den von ihr durch Anwenden des Spannungsoperators ableitbaren Tensoren sind dieselben, wie bei den entsprechenden Ausdrücken der klassischen Potentialtheorie der Laplace Gleichung; viele Aussagen über die Regulariätseigenschaften der hydrodynamischen Potentiale lassen sich dementsprechend ähnlich dem klassischen Fall der Laplace-Gleichung machen. Die Beweise zu den in diesem Abschnitt ohne Beweis formulierten Sätze kann man z. B. in [Lad] nachschlagen. 
Eine kleine Bemerkung zu den in dieser Arbeit verwendeten Bezeichungen: anstelle der etwas umständlichen Raumbezeichnungen $\left(L^{2}(D)\right)^{3},(C(D))^{2}$ u.ä. wird einfach $L^{2}(D), C(D)$ usw. geschrieben, solange die gemeinte Bedeutung aus dem Kontext eindeutig hervorgeht.

Sei $\partial D$ Rand eines beschränkten zusammenhängenden Gebiets D. Das Einfachschichtpotential (ESP) einer Dichte $\varphi \in H^{-\frac{1}{2}}(\partial D)$, gegeben für $x \in \mathbb{R}^{m} \backslash \partial D$ durch das Integral

$$
\left(\boldsymbol{S}_{m} \varphi\right)(x):=\int_{\partial D} \Psi^{(c)}(x-y) \varphi(y) d s(y),
$$

entspricht einem Fluß, der durch auf der Fläche $\partial D$ gemäß $\varphi$ verteilte Punktkräfte erzeugt wird. Es löst die Stokes-Gleichung für alle Punkte in $\mathbb{R}^{m} \backslash \partial D$.

Zwar wird der Kern des Einfachschichtpotentials singulär für Punkte der Fläche $\partial D$, aber die entsprechenden Integrale existieren als uneigentliche, wobei gilt:

\section{Satz 2.2}

Ist $\partial D$ eine Lyapunov-Fläche und die Dichte $\varphi$ stetig, so ist das Geschwindigkeitsfeld des Einfachschichtpotentials, gegeben durch

$$
\left(S_{m} \varphi\right)(x):=\int_{\partial D} \Psi^{(r, c)}(x-y) \varphi(y) d s(y), \quad x \in \mathbb{R}^{m}
$$

im ganzen $\mathbb{R}^{m}$ stetig.

Unter dem Doppelschichtpotential (DSP) einer Dichte $\varphi \in C(\partial D)$ bzw. $L^{2}(\partial D)$ versteht man das $m+1$-dimensionale Vektorfeld

$$
\left(\boldsymbol{K}_{m} \varphi\right)(x):=\int_{\partial D} k_{m}(x, y) \varphi(y) d s(y) \quad x \in \mathbb{R}^{m} \backslash \partial D,
$$

mit dem $(m+1) \times m$ Kern

$$
k_{m}(x, y)=\left(\left(T_{x} \Psi_{l}(x-y)\right)_{i j} \nu_{j}(y)\right)_{l i},
$$

welcher für $i, l=1, \ldots, m, m \geq 2$ durch

$$
\begin{array}{r}
k_{m ; l i}(x, y)=-\frac{m}{\omega_{m}} \frac{z_{l} z_{i} z \cdot \nu(y)}{|z|^{m+2}}, \\
k_{m ; m+1, i}(x, y)=-\frac{2}{\omega_{m}}\left(\frac{z_{i} z \cdot \nu(y)}{|z|^{m+2}}-\frac{\nu_{i}(y)}{|z|^{m}}\right)
\end{array}
$$

mit $z:=x-y$ explizit gegeben ist.

Auch das Doppelschichtpotential löst die Stokes-Gleichung in $\mathbb{R}^{m} \backslash \partial D$. 
Das Vektorfeld $K_{m} \varphi$, gegeben durch

$$
\left(K_{m} \varphi\right)(x):=\int_{\partial D} k_{m}^{(r)}(x, y) \varphi(y) d s(y), \quad x \in \mathbb{R}^{m} \backslash \partial D,
$$

entspricht der Geschwindigkeitskomponente des DSP.

Desweiteren ist die normale Spannung $-T\left(\boldsymbol{S}_{\boldsymbol{m}} \varphi\right) \nu$ des Einfachschichtpotentials von Interesse. Ist für die Fläche $\partial D$ die Konstruktion von Parallelflächen möglich, so ist diese bei stetigen Dichten $\varphi$ in einer Umgebung $U$ von $\partial D$ durch

$$
\begin{array}{r}
\left(K_{m}^{\prime} \varphi\right)(x)=-\int_{\partial D} T_{x}\left(\Psi^{(c)}(x-y) \varphi(y)\right) \nu(\tilde{x}) d s(y) \\
=: \int_{\partial D} H(x, y) \varphi(y) d s(y), \quad x \notin \partial D
\end{array}
$$

gegeben, wobei mit $\tilde{x}$ die eindeutig bestimmte Projektion von $x \in U \backslash \partial D$ auf $\partial D$ bezeichnet ist.

Die Integrale 2.7 und 2.8 existieren auch für $x \in \partial D$ (s. [Lad]); für die dadurch definierte Direktwerte von $K_{m} \varphi$ und $K_{m}^{\prime} \varphi$ auf $\partial D$ verwenden wir die Bezeichnungen $(K \varphi)_{(\partial D)}$ und $\left(K^{\prime} \varphi\right)_{(\partial D)}$. Da für die Matrix $H$ in 2.8 die Beziehung

$$
H(x, y)=\left(k_{m}^{(r)}(x, y)\right)^{T}=k_{m}^{(r)}(y, x), \quad x, y \in \partial D
$$

gilt (vgl. VVarnhorn], S. 18), sind die Randintegraloperatoren $K_{(\partial D)}$ und $K_{(\partial D)}^{\prime}$ bezüglich des Dualsystems

$$
<\varphi, \psi>:=\int_{\partial D} \varphi \cdot \psi d s
$$

zueinander adjungiert.

Für die Vektorfelder $\left(K_{m} \varphi\right)$ und $\left(K_{m}^{\prime} \varphi\right)$ gelten dem potentialtheoretischen Fall ähnliche Sprungrelationen:

\section{Satz 2.3}

Ist $\partial D$ eine Lyapunov-Fläche, so sind die Vektorfelder $K_{m}$ und $K_{m}^{\prime}$ bei Dichten $\varphi \in C(\partial D)$ stetig in $D$ und in $\mathbb{R}^{m} \backslash \bar{D}$, wobei für ihre Grenzwerte an $\partial D$ gilt

$$
(K \varphi)_{ \pm}=\mp \frac{1}{2} \varphi+(K \varphi)_{(\partial D)}
$$

und

$$
\left(K^{\prime} \varphi\right)_{ \pm}= \pm \frac{1}{2} \varphi+\left(K^{\prime} \varphi\right)_{(\partial D)}
$$




\section{Anmerkung}

Ist das Paar $(u, p)$ das Einfachschichtpotential einer stetigen Belegung $\varphi$, so kann man die Sprungbeziehung 2.10 in der Form

$$
\left(T_{p}^{u} \nu\right)_{ \pm}=\mp \frac{1}{2} \varphi+\left(T_{p}^{u} \nu\right)_{(\partial D)}
$$

schreiben.

Klar von den Potentialen selbst sind die folgenden Randintegraloperatoren zu unterscheiden, die wir hier für das Weitere einführen:

$$
\begin{aligned}
(S \varphi)_{(\partial D)}(x) & =\int_{\partial D} \Psi^{(r, c)}(x-y) \varphi(y) d s(y), \quad x \in \partial D \\
(K \varphi)_{(\partial D)}(x) & =\int_{\partial D} k_{m}^{(r)}(x, y) \varphi(y) d s(y), \quad x \in \partial D \\
\left(K^{\prime} \varphi\right)_{(\partial D)}(x) & =-\int_{\partial D} T_{x}\left(\Psi^{(c)}(x-y) \varphi(y)\right) \nu(x) d s(y), \quad x \in \partial D
\end{aligned}
$$

Um im weiteren die Lösbarkeitstheorie für das Äussere Dirichlet Problem bei $L^{2}$ Randdaten zu entwickeln, brauchen wir die Erweiterung der Aussagen der Sätze 2.2 und 2.3 für quadratsummierbare Dichten. Aussagen dieser Art wurden für die Potentiale bei der Helmholtz-Gleichung von H. Kersten ([Kersten]) gezeigt. Die Idee seines Beweises kann ohne weiteres auf die Stokes-Potentiale übertragen werden; da dies in der Literatur noch nicht explizit gemacht worden ist, erscheint es uns ratsam, hier einen Beweis zu geben.

\section{Lemma 2.4}

Ist $\partial D C^{2}$-glatt und $\varphi \in L^{2}(\partial D)$, so gilt

$$
\begin{array}{ll}
\lim _{s \rightarrow 0_{+}} & \int_{\partial D}\left|(S \varphi)(x \pm s \nu(x))-(S \varphi)_{(\partial D)}(x)\right|^{2} d s(x) \rightarrow 0 \\
\lim _{s \rightarrow 0_{+}} \int_{\partial D}\left|(K \varphi)(x \pm s \nu(x))-(K \varphi)_{(\partial D)}(x) \pm \frac{1}{2} \varphi(x)\right|^{2} d s(x) \rightarrow 0 \\
\lim _{s \rightarrow 0_{+}} \int_{\partial D}\left|\left(K^{\prime} \varphi\right)(x \pm s \nu(x))-\left(K^{\prime} \varphi\right)_{(\partial D)}(x) \mp \frac{1}{2} \varphi(x)\right|^{2} d s(x) \rightarrow 0
\end{array}
$$

Beweis: Für $s>0$ betrachte man Systeme $S_{s}, K_{s}, K_{s}^{\prime}$ von aus $L^{2}(\partial D)$ nach $C(\partial D)$ wirkenden Operatoren, gegeben durch

$$
\begin{aligned}
\left(S_{s} \varphi\right)(x) & :=(S \varphi)(x+s \nu(x)) \\
\left(K_{s} \varphi\right)(x) & :=(K \varphi)(x+s \nu(x)) \\
\left(K_{s}^{\prime} \varphi\right)(x) & :=\left(K^{\prime} \varphi\right)(x+s \nu(x))
\end{aligned}
$$

Als Operatoren mit stetigen, bei $s=0$ ggf. schwach singulären Kernen sind $S_{s}, K_{s}$ und $K_{s}^{\prime}$ für alle $s \geq 0$ stetig bezüglich der $L^{2}$-Norm, eingeschränkt auf $C(\partial D)$ auch 
bezüglich der Maximumnorm.

Führt man nun für $s>0$ die Operatoren

$$
\begin{aligned}
S_{s, 0} & :=S_{ \pm s}-S_{0}, \\
K_{s, 0} & :=K_{ \pm s}-K_{0} \pm \frac{1}{2} I, \\
K_{s, 0}^{\prime} & :=K^{\prime} \pm s-K_{0}^{\prime} \mp \frac{1}{2} I
\end{aligned}
$$

ein, so können die Aussagen der Sätze 2.2 und 2.3 folgendermaßen zusammengefasst werden: für $\varphi \in C(\partial D)$ gilt

$$
\lim _{s \rightarrow 0_{+}}\left\|S_{s, 0} \varphi\right\|_{\infty}=\lim _{s \rightarrow 0_{+}}\left\|K_{s, 0} \varphi\right\|_{\infty}=\lim _{s \rightarrow 0_{+}}\left\|K_{s, 0}^{\prime} \varphi\right\|_{\infty}=0 .
$$

Das gleiche gilt auch für die $L^{2}$-adjungierten Operatoren $S_{s, 0}^{*}, K_{s, 0}^{*}, K_{s, 0}^{\prime *}$ : da diese sich durch das formale Vertauschen der Argumente der jeweiligen Integralkerne ergeben, erfolgt die Herleitung der Beziehungen

$$
\lim _{s \rightarrow 0_{+}}\left\|S_{s, 0}^{*} \varphi\right\|_{\infty}=\lim _{s \rightarrow 0_{+}}\left\|K_{s, 0}^{*} \varphi\right\|_{\infty}=\lim _{s \rightarrow 0_{+}}\left\|K_{s, 0}^{\prime *} \varphi\right\|_{\infty}=0
$$

bei $\varphi \in C(\partial D)$ analog zu 2.18 .

Sei $\left(T_{s}\right)_{0<s \leq s_{0}}$ irgend eines der Operatorensysteme $S_{s}, K_{s}, K_{s}^{\prime}$. In der schwächeren $L^{2}$-Norm gilt also für $\varphi \in C(\partial D)$

$$
\lim _{s \rightarrow 0_{+}}\left\|T_{s} \varphi\right\|_{L^{2}(\partial D)}=\lim _{s \rightarrow 0_{+}}\left\|T_{s}^{*} \varphi\right\|_{L^{2}(\partial D)}=0,
$$

und es bietet sich an, den Satz von Banach-Steinhaus anzuwenden, um 2.20 auch für $\varphi \in L^{2}(\partial D)$ zu folgern. Dafür muß die gleichmäßige Beschränktheit der Operatoren $\left(T_{s}\right)_{0<s \leq s_{0}}$ in $L^{2}(\partial D)$ gezeigt werden.

Aus 2.18 und 2.19 folgt, daß für jedes $\varphi \in C(\partial D)$ eine Konstante $c_{\varphi}$ mit $\left\|T_{s} \varphi\right\|_{\infty}<c_{\varphi}$ und $\left\|T_{s}^{*} \varphi\right\|_{\infty}<c_{\varphi}$ für alle $s$ existiert. Das Prinzip der gleichmäßigen Beschränktheit, angewandt im Banachraum $\left(C(\partial D),\|\cdot\|_{\infty}\right)$, sichert nun die Existenz einer Konstanten $C$ mit $\left\|T_{s} \mid C(\partial D)\right\|_{\infty} \leq C$ und $\left\|T_{s}^{*} \mid C(\partial D)\right\|_{\infty} \leq C$ für alle $s$.

Für die selbstadjungierten und in $\left(C(\partial D),\|\cdot\|_{\infty}\right)$ stetigen Operatoren $T_{s}^{*} T_{s}$ liefert nun der Satz von Lax

$$
\left\|T_{s}^{*} T_{s}\right\|_{L^{2}(\partial D)} \leq\left\|T_{s}^{*} T_{s} \mid C(\partial D)\right\|_{\infty}
$$

woraus mit der Identität $\left\|T_{s}\right\|_{L^{2}(\partial D)}^{2}=\left\|T_{s}^{*}\right\|_{L^{2}(\partial D)}^{2}=\left\|T_{s}^{*} T_{s}\right\|_{L^{2}(\partial D)}$ und der obigen Konstanten $C$ folgt

$$
\left\|T_{s}\right\|_{L^{2}(\partial D)}=\left\|T_{s}^{*}\right\|_{L^{2}(\partial D)} \leq C, \quad \forall s \in\left(0, s_{0}\right)
$$


und die Aussage des Lemmas folgt mit Anwendung des Satzes von Banach-Steinhaus. 


\section{2 Äusseres Dirichlet Problem bei $C^{1}$-Randdaten}

Sei $D \subset \mathbb{R}^{m}, m=2,3$ ein beschränktes zusammenhängendes Gebiet mit geschlossenem $C^{2}$-Rand $\partial D$. Wir betrachten zunächst das äussere Dirichlet Problem für das Stokessche System in dessen klassischer Formulierung. Diese besteht in der Aufgabe, ein Lösungspaar $(u, p)$ mit

$$
u \in\left(C^{2}\left(D^{+}\right) \cap C^{1}\left(\overline{D^{+}}\right)\right)^{2}, p \in C^{1}\left(D^{+}\right) \cap C\left(\overline{D^{+}}\right)
$$

$\mathrm{zu}$

$$
\left\{\begin{array}{c}
-\Delta u+\operatorname{grad} p=0 \\
\operatorname{div} u=0
\end{array} \quad \text { in } D^{+},\right.
$$

zu finden, wobei $u$, eingeschränkt auf den Rand des Gebiets, mit einem vorgegebenen Vektorfeld $f \in C^{1}(\partial D)$ übereinstimmt und im Unendlichen folgendes Verhalten aufweist:

$$
\begin{aligned}
\nabla^{k} u(x) & =O\left(|x|^{2-m-k}\right), k=0,1, \\
p(x) & =O\left(|x|^{1-m}\right) .
\end{aligned}
$$

Mit $D^{+}$wird hier, wie üblich, das unbeschränkte Gebiet $D^{+}:=\mathbb{R}^{m} \backslash \bar{D}$ bezeichnet.

Der Nachweis der Eindeutigkeit für dieses Problem stellt wegen der Anwendbarkeit der Greenschen Formel keine Schwierigkeit dar; es gilt (siehe Varnhorn, Lemma 1.1):

\section{Satz 2.5}

Das ÄDP 2.21 2.24 hat für beliebige Randaten $f \in C^{1}(\partial D)$ höchstens eine Lösung. Ist ein $a_{\infty} \in \mathbb{R}^{2}$ vorgegeben, kann die Bedingung 2.23 im Fall $m=2, k=0 z u$

$$
u(x)-a_{\infty} \ln |x|=O(1)
$$

ohne Beeinträchtigung der Eindeutigkeit abgeschwächt werden.

Das innere Dirichlet Problem, dessen Formulierung auf natürliche Weise aus der obigen Definition durch das Ersetzen von $D^{+}$mit $D$ und die Wegnahme der Asymptotikanforderung hervorgeht, steht nicht im Mittelpunkt unserer Untersuchungen; für technische Zwecke brauchen wir jedoch seine Eindeutigkeit. Zugleich zeigt die folgende Aussage einen anderen Weg zum Eindeutigkeitsbeweis sowie eine leichte Abschwächung der Regularitätsanforderungen des Satzes 2.5.

\section{Satz 2.6}

Das innere Dirichlet Problem für Randdaten $f \in H^{\frac{1}{2}}(\partial D)$ mit

$$
\int_{\partial D} f \cdot \nu d s=0
$$


und das äußere Dirichlet Problem für Randdaten $f \in H^{\frac{1}{2}}(\partial D)$ sind in $H^{1}(D)$ bzw. $W^{1}\left(D^{+}\right):=\left\{u: \frac{u(x)}{\left(1+|x|^{2}\right)^{\frac{1}{2}}} \in L^{2}\left(D^{+}\right), \frac{\partial u}{\partial x_{i}} \in L^{2}\left(D^{+}\right), i=1, \ldots, m\right\}$ eindeutig lösbar.

Beweis: Wir skizzieren hier den in Dautray/Lions] gegebenen Beweis. Nach Lad] existiert zu den Randdaten $f$ eine Erweiterungsabbildung $R f \in H^{1}(D)$ beziehungsweise $R f \in W^{1}\left(D^{+}\right)$mit

$$
\begin{aligned}
& \left.R f\right|_{\partial D}=f \text { und } \\
& \operatorname{div}(R f)=0 \text { in } D \text { bzw. } D^{+} .
\end{aligned}
$$

Mit der Bezeichung $H_{0}^{1}(D)$ für den Abschluß von $C_{0}^{\infty}(D)$ in $H^{1}(D)$ sowie $W_{0}^{1}\left(D^{+}\right)$ für den Abschluß von $C_{0}^{\infty}\left(D^{+}\right)$in $W^{1}\left(D^{+}\right)$kann man nun das innere und das äußere Dirichlet Problem in folgende Variationsformulierungen für die Unbekannte $v:=$ $u-R f, f \in H^{\frac{1}{2}}(\partial D)$ überführen:

$$
\begin{aligned}
& \int_{D \text { bzw. } D^{+}} D v: D w d x=-\int_{D \text { bzw. } D^{+}} D(R f): D w d x \\
& \operatorname{div} v=0, \\
& v \in H_{0}^{1}(D) \quad \text { bzw. } \quad \in W_{0}^{1}\left(D^{+}\right)
\end{aligned}
$$

für alle $w \in H_{0}^{1}(D)$ bzw. $w \in W_{0}^{1}(D)$ mit $\operatorname{div} w=0$.

Diese Probleme lassen sich mit dem Satz von Lax-Milgram behandeln. Die Koerzivität der Bilinearform der linken Seite in 2.25 für das innere Problem folgt aus der Ungleichung von Poincaré, die für die linke Seite beim äußeren Problem aus einer geeigneten Darstellung der Norm in $W_{0}^{1}\left(D^{+}\right)$(s. Dautray/Lions]).

Die Existenz von Lösungen zum Problem 2.21 2.24 kann auf verschiedenen Wegen gezeigt werden. Zum Einen besteht die Möglichkeit, den Einfachschichtpotentialansatz anzuwenden, der zur Lösung einer Integralgleichung erster Art mit dem kompakten Operator $S_{\partial D}$ führt, was mit klaren numerischen Nachteilen verbunden ist. Sucht man im zweidimensionalen Fall die beschränkte Lösung des ÄDP, muß der Einfachschichtpotentialansatz wegen des logarithmischen Verhaltens der Grundlösung im Unendlichen zusätzlich modifiziert werden (vgl. [Kress1, S. 118f).

Der Wunsch, zur konstruktiver Lösung der Dirichlet Probleme zum Stokesschen System Integralgleichungen zweiter Art zu erhalten, führt zum Gebrauch des Doppelschichtpotentials im Ansatz. Da dieses allein nicht ausreicht, um beliebige StokesFlüsse darzustellen (wie der folgende Satz es zeigt), wurden in der Literatur einige Abänderungen des Doppelschichtpotentialansatzes vorgeschlagen. Wir benutzen sie sowohl für die numerische Behandlung des Vorwärtsproblems, als auch für die Theorie des inversen Problems. 


\section{Satz 2.7}

Die Nullräume der Operatoren $\frac{1}{2} I+K$ und $\frac{1}{2} I+K^{\prime}$ sind eindimensional und sind gegeben durch

$$
N\left(\frac{1}{2} I+K\right)=\operatorname{span}\left\{\psi_{0}\right\}, \quad N\left(\frac{1}{2} I+K^{\prime}\right)=\operatorname{span}\{\nu\}
$$

mit einem $\psi_{0}$, für das gilt

$$
\int_{\partial D} \psi_{0} \cdot \nu d s \neq 0
$$

Die Nullräume der Operatoren $\frac{1}{2} I-K$ and $\frac{1}{2} I-K^{\prime}$ haben Dimension 6 im räumlichen und $3 \mathrm{im}$ zweidimensionalen Fall.

Beweis: Siehe z.B. Power/Wrobel]. Von Odqvist (1938) wurde gezeigt, daß die homogene Integralgleichung $\frac{1}{2} \varphi-K^{\prime} \varphi=0$ im $\mathbb{R}^{m}$ genau sechs $(m=3)$ bzw. drei $(m=2)$ linear unabhängige Lösungen besitzt, nämlich die Vektoren der Starrkörperbewegungen im $\mathbb{R}^{m}$. Diese sind im Zweidimensionalen durch $\varphi_{i}=e_{i}, i=1,2$ (die Einheitsvektoren) und $\varphi_{3}=\left(x_{2},-x_{1}\right)$ gegeben (wir nehmen o.B.d.A. an, daß der Koordinatenanfang im Gebiet $D$ liegt).

Der Idee, das Spektrum von $K_{\partial D}$ durch zusätzliche Terme zu stören, um diese Singularität seiner Resolvente zu beseitigen, ist die Methode des gemischten Doppelund Einfachschichtpotentialansatzes entsprungen. Sie wurde eingeführt für den Fall $m=2$ in (

\section{Satz 2.8}

Für jedes $a_{\infty} \in \mathbb{R}^{2}$ und $f \in C(\partial D)$ ist die Integralgleichung

$$
f+\frac{4 \pi}{|\partial D|} S_{2} a_{\infty}=\left(-\frac{1}{2} I+K_{(\partial D)}-\eta S_{(\partial D)}(I-M)-\alpha|\partial D| M\right) \varphi
$$

mit dem Oberflächenmittelwert $M$

$$
M \varphi:=\frac{1}{|\partial D|} \int_{\partial D} \varphi(y) d s,
$$

und einem Parameterpaar $\eta>0, \alpha \neq 0$ eindeutig lösbar.

Ist $\varphi \in C(\partial D)$ Lösung von 2.29, so ist die Lösung des äußeren Stokes Problems durch

$$
\begin{aligned}
\left(\begin{array}{l}
u \\
p
\end{array}\right)(x)=-\frac{4 \pi}{|\partial D|}\left(\boldsymbol{S}_{\mathbf{2}} a_{\infty}\right)(x) & +\left(\boldsymbol{K}_{\mathbf{2}} \varphi\right)(x) \\
& -\eta\left(\boldsymbol{S}_{\mathbf{2}}\left(I-M_{2}\right) \varphi\right)(x)-\alpha \int_{\partial D}\left(\begin{array}{l}
\varphi \\
0
\end{array}\right) d s
\end{aligned}
$$

gegeben. Durch geeignete Wahl der Parameter $\alpha, \eta$ läßt sich die Konditionszahl entsprechender Integraloperatoren mininieren. 


\section{Anmerkung 2.1}

Auf diesem Ansatz basiert die von uns verwendete numerische Methode zur Behandlung des äußeren Dirichlet Problems. Man beachte die Modifikation des Einfachschichtpotentials, die im Ansatz verwendet ist: wegen des erwähnten logarithmischen Verhaltens der Grundlösung im Unendlichen ist sie notwendig, um den Fall $a_{\infty}=0$ nicht auszuschliessen.

Die Erweiterung der Methode der gemischten Potentiale auf den dreidimensionalen Fall findet man in Hebeker:

\section{Satz 2.9}

Der Operator $-\frac{1}{2} I+K_{(\partial D)}-S_{(\partial D)}: C(\partial D) \rightarrow C(\partial D)$ ist injektiv.

Der Ansatz

$$
\left(\begin{array}{l}
u \\
p
\end{array}\right)(x)=\left(\boldsymbol{K}_{3} \varphi\right)(x)-\left(\boldsymbol{S}_{\mathbf{3}} \varphi\right)(x),
$$

liefert die Lösung des Problems 2.21,2.24, falls die Funktion $\varphi$ die Randintegralgleichung

$$
f=\left(-\frac{1}{2} I+K_{(\partial D)}-S_{(\partial D)}\right) \varphi
$$

erfüllt.

In einem nachfolgendem Abschnitt geben wir eine kurze Beschreibung des NyströmVerfahrens zur numerischen Behandlung der Integralgleichung 2.29. Dabei werden wir sehen, daß die Voraussetzung eines $C^{2}$-glatten Randes auch für die Numerik wesentlich ist: die Approximation der hier vorkommenden Integrale erfordert wegen der Singularität im Kern des Einfachschichtpotententials eine Taylorentwicklung des Integranden in einer Umgebung des singulären Punktes, wobei Terme zweiter Ordnung vorkommen.

Vor allem, um dieses zu umgehen, haben Power and Miranda ([Power/Miranda ) gezeigt, daß man die Lücke im Wertebereich des Doppelschichtpotentials schliessen kann, indem das Einfachschichtpotential in 2.32 durch eine Konstante und ein Paar sich im Gebiet befindenden Singularitäten, nämlich durch eine Kombination eines Stokelets gegebener Stärke und eines Stokesschen Rotlets mit Druck gleich Null und einer unbekannter Stärke, ersetzt werden kann².

Diese Methode, in der Literatur als the completed double layer boundary integral method bekannt, wurde in ([Power $]$ ) auf den zweidimensionalen Fall übertragen. Für unsere Anwendungen, nämlich für die theoretische Behandlung des inversen Problems im Zweidimensionalen, ist die damit verbundene Lösungsdarstellung insofern

\footnotetext{
${ }^{2}$ Das Stokessche Rotlet konstanter Stärke $w$ ist definiert als die singuläre Lösung der inhomogenen Stokes-Gleichung mit $\nabla x w \delta(x)$ in der rechten Seite.
} 
interessant, daß sie das im Unendlichen im Allgemeinen nicht abklingende Einfachschichtpotential nicht beinhaltet.

Entsprechend der Formulierung in Power sucht man das Geschwindigkeitsfeld in der Form

$$
\begin{aligned}
u(x)=\Psi^{(r, c)}(x) a_{\infty} & +\left(K_{2} \varphi\right)(x)+ \\
& +\frac{1}{2 \pi|x|^{2}}\left(\begin{array}{c}
-x_{2} \\
x_{1}
\end{array}\right) \int_{\partial D} \varphi(y) \cdot\left(\begin{array}{c}
y_{2} \\
-y_{1}
\end{array}\right) d s(y)-\frac{|\partial D|}{2 \pi} M \varphi
\end{aligned}
$$

mit gewisser Dichtefunktion $\varphi \in C(\partial D)$.

Das zweidimensionale Rotlet der Stärke $w$ hat die Gestalt

$$
\left(R_{i} \varphi\right)(x)=\frac{\epsilon_{i j k} w_{j}(\varphi) \boldsymbol{x}_{k}}{|x|^{2}} \quad i=1,2 ; j, k=1,2,3,
$$

wobei $\boldsymbol{x}$ als dreidimensionaler Vektor $\boldsymbol{x}=\left(x_{1}, x_{2}, 0\right)$ definiert ist und $\epsilon_{i j k}$ den alternierenden Einheitstensor bezeichnet. Der Term

$$
(L \varphi)(x):=\frac{1}{2 \pi|x|^{2}}\left(\begin{array}{c}
-x_{2} \\
x_{1}
\end{array}\right) \int_{\partial D} \varphi(y) \cdot\left(\begin{array}{c}
-y_{2} \\
y_{1}
\end{array}\right) d s(y)
$$

im Ansatz stellt also ein Rotlet mit

$$
w_{1}=w_{2}=0, \quad w_{3}=\frac{1}{2 \pi} \int_{\partial D} \varphi(x) \cdot\left(\begin{array}{c}
-x_{2} \\
x_{1}
\end{array}\right) d s(x)
$$

dar; man merke, daß $\left(x_{2},-x_{1}\right)$ eine der Festkörperbewegungen der zweidimensionalen Flüssigkeit darstellt. Der Term $M \varphi$ ist in seinen Komponenten linear von den beiden anderen Festkörperbewegungen $(1,0)$ und $(0,1)$ abhängig. Dadurch läßt sich die Lücke im Wertebereich des Doppelschichtpotentialoperators kompensieren. Es gilt ([Power $])$

Satz 2.10

Die dem Ansatz 2.34 entsprechende Randintegralgleichung

$$
f-\Psi^{(r, c)} a_{\infty}=-\frac{1}{2} \varphi+K_{2} \varphi+L \varphi-\frac{|\partial D|}{2 \pi} M \varphi
$$

ist in $C(\partial D)$ eindeutig lösbar. Ist das Vektorfeld $\varphi$ Lösung dieser Gleichung, so liefert der Ansatz 2.34 die eindeutige Lösung des ÄDP 2.21.2.24. 


\section{3 Äusseres Dirichlet Problem bei $L^{2}$-Randdaten}

In diesem Abschnitt wollen wir die für die Behandlung des inversen Problems notwendigen Resultate der Lösungstheorie auf den Fall quadratsummierbarer Randdaten erweitern. Vor allem soll die Eindeutigkeit des ÄDP auch bei schwächerer Randregularität von $u$ nachgewiesen werden.

Unsere Vorgehensweise beim Eindeutigkeitsnachweis ist von [Hähner2] inspiriert; die Grundidee der Verwendung von Parallelflächen zur Feststellung der Eindeutigkeit eines Randwertproblems bei $L^{2}$-Randdaten stammt von Calderón (vgl. [Calderón]).

\section{Satz 2.11}

Sei $u \in C^{2}\left(D^{+}\right)$die Geschwindigkeitskomponente einer Lösung der Stokes-Gleichung in $D^{+}$, die die homogene Randbedingung

$$
\int_{\partial D} u(x+h \nu(x)) g(x) d s(x) \rightarrow 0, h \rightarrow 0
$$

für alle $g \in L^{2}(\partial D)$ erfüllt und sich im Unendlichen gemäß 2.23 verhält.

Ist $D C^{3}$-regulär, so gilt: $u=0$ in $D^{+}$.

Beweis:

Für hinreichend kleine $h$ seien Parallelflächen

$$
\partial D_{h}:=\left\{z \in \mathbb{R}^{3}: z=x+h \nu(x), x \in \partial D\right\}
$$

definiert. Über diese ist Folgendes bekannt:

Ist für $x \in \partial D \hat{H}(x)$ die mittlere Krümmung und $\hat{K}(x)$ die Gaußsche Krümmung der Fläche $\partial D$ bei $x$, so ist das infinitesimale Flächenelement von $\partial D_{h}$ um $z=x+h \nu(x)$ mit dem Flächenelement von $\partial D$ im Punkt $x$ durch die Relation

$$
d s(z)=\left(1-2 \hat{H}(x) h+\hat{K}(x) h^{2}\right) d s(x)
$$

verbunden. Da uns hier nur äussere Parallelflächen interessieren, sei $h>0$ im Weiteren immer vorausgesetzt.

Für jedes $h>0$ betrachte man das Dirichlet Problem im Gebiet $D_{h}^{+}$mit den Randdaten $\left.u\right|_{\partial D_{h}}$. Die Eindeutigkeit und die Existenz jeweiliger Lösungen $v_{h}$ sind durch die Sätze 2.5 und 2.9 gesichert, somit gilt einerseits $\left.u\right|_{D_{h}^{+}} \equiv v_{h}$ und andererseits die Darstellbarkeit von $v_{h}$ als Potential (vgl. 2.32)

$$
v_{h}(x):=\left(K_{\partial D_{h}} \tilde{\varphi}_{h}\right)(x)-\eta\left(S_{\partial D_{h}} \tilde{\varphi}_{h}\right)(x),
$$

einer Belegung $\tilde{\varphi}_{h} \in C^{1, \alpha}\left(\partial D_{h}\right)$, welche als die eindeutige Lösung der Integralgleichung

$$
-\frac{1}{2} \tilde{\varphi}_{h}(\tilde{x})+\left(K_{\partial D_{h}} \tilde{\varphi}_{h}\right)(\tilde{x})-\eta\left(S_{\partial D_{h}} \tilde{\varphi}_{h}\right)(\tilde{x})=u(\tilde{x}), \tilde{x} \in \partial D_{h}
$$


ermittelbar ist.

Führt man nun den Homöomorphismus $\alpha_{h}: C(\partial D) \rightarrow C\left(\partial D_{h}\right)$ mit

$$
\left(\alpha_{h} f\right)(x+h \nu(x)):=f(x), x \in \partial D
$$

ein, so erhält man äquivalent zu 2.39 die Gleichung

$$
\left(-\frac{1}{2} I+K_{h}-\eta S_{h}\right) \varphi_{h}=\alpha_{h}^{-1}\left(\left.u\right|_{\partial D_{h}}\right)
$$

mit $\varphi_{h}:=\alpha^{-1} \tilde{\varphi}_{h}$, wobei die Operatoren $K_{h}$ und $S_{h}$ für $\varphi \in C(\partial D), x \in \partial D$ durch

$$
\left(K_{h} \varphi\right)_{i}(x):=-\frac{3}{4 \pi} \int_{\partial D} \frac{z_{k} z_{i} z \cdot \nu(y)}{|z|^{m+2}} \varphi(y)\left(1-2 \hat{H}(y) h+\hat{K}(y) h^{2}\right) d s(y) \quad i, k=1,2,3
$$

mit $z:=x-y+h(\nu(x)-\nu(y)$ und

$$
\left(S_{h} \varphi\right)(x):=\int_{\partial D} \Psi^{(r, c)}(x+h \nu(x), y+h \nu(y)) \varphi(y)\left(1-2 \hat{H}(y) h+\hat{K}(y) h^{2}\right) d s(y)
$$

gegeben sind.

Nun überzeugen wir uns, daß die Lösungen der Integralgleichung 2.40 bei $h \rightarrow 0$ schwach gegen Null konvergieren.

Die Kerne $k(x, y, h)$ und $s(x, y, h)$ der Operatoren $K_{h}$ und $S_{h}$ sind in $x$ und $y$ stetig für $x \neq y$ und schwach singulär. Darüber hinaus folgt direkt aus dem Mittelwertsatz, daß für $|x-y|>\delta$ bei jedem festen $\delta$ die Elemente der Funktionalmatrizen $k(x, y, h)$ und $s(x, y, h)$ gleichmäßig gegen die entsprechenden Elemente von $k(x, y, 0)$ bzw. $s(x, y, 0)$ konvergieren. Somit sind die Bedingungen des Hilfsatzes A1 in [Hähner2] erfüllt und es gilt:

$$
\left\|K_{h}-\eta S_{h}-(K-\eta S)\right\|_{L^{2}(\partial D)} \rightarrow 0, h \rightarrow 0 .
$$

Für hinreichend kleine $h$ liefert nun die Neumannsche Reihe

$$
\left\|\left(-\frac{1}{2} I+K_{h}-\eta S_{h}\right)^{-1}-\left(-\frac{1}{2} I+K-\eta S\right)^{-1}\right\|_{L^{2}(\partial D)} \rightarrow 0, h \rightarrow 0 .
$$

Die Voraussetzung 2.37 garantiert die schwache Konvergenz von $\left(\left.\alpha^{-1} u\right|_{\partial D_{h}}\right)$ gegen 0 im $L^{2}$-Sinne, somit gibt es für alle $h \in\left(0, h_{0}\right), h_{0}$ ausreichend klein, eine Konstante $C$ mit $\left\|\left(\left.\alpha^{-1} u\right|_{\partial D_{h}}\right)\right\|_{L^{2}(\partial D)}<C$. Insgesamt erhalten wir:

$$
\begin{aligned}
\varphi_{h} & =\left(-\frac{1}{2} I+K_{h}-\eta S_{h}\right)^{-1}\left(\left.\alpha^{-1} u\right|_{\partial D_{h}}\right) \\
& =\left\{\left(-\frac{1}{2} I+K_{h}-\eta S_{h}\right)^{-1}-\left(-\frac{1}{2} I+K-\eta S\right)^{-1}\right\}\left(\left.\alpha^{-1} u\right|_{\partial D_{h}}\right) \\
& +\left(-\frac{1}{2} I+K-\eta S\right)^{-1}\left(\left.\alpha^{-1} u\right|_{\partial D_{h}}\right) .
\end{aligned}
$$


Da für die Summanden dieser Geleichung

$$
\begin{array}{r}
\left\|\left\{\left(-\frac{1}{2} I+K_{h}-\eta S_{h}\right)^{-1}-\left(-\frac{1}{2} I+K-\eta S\right)^{-1}\right\}\left(\left.\alpha^{-1} u\right|_{\partial D_{h}}\right)\right\| \\
\leq C\left\|\left\{\left(-\frac{1}{2} I+K_{h}-\eta S_{h}\right)^{-1}-\left(-\frac{1}{2} I+K-\eta S\right)^{-1}\right\}\right\|_{L^{2}(\partial D)} \rightarrow 0,
\end{array}
$$

und

$$
\left(-\frac{1}{2} I+K-\eta S\right)^{-1}\left(\left.\alpha^{-1} u\right|_{\partial D_{h}}\right) \rightarrow 0, h \rightarrow 0, h>0 .
$$

gilt, erhält man $\varphi_{h} \rightarrow 0, h \rightarrow 0$ und demzufolge $\tilde{\varphi}_{h} \rightarrow 0, h \rightarrow 0$.

Für einen beliebigen Punkt $x \in D^{+}$konvergieren folglich die Werte der Ansatzfunktion $v_{h}$ mit $h \rightarrow 0$ gegen 0 , d.h. es gilt $u(x)=0$ für alle $x \in D^{+}$.

\section{Anmerkung 2.2}

Die Voraussetzung eines $C^{3}$-glatten Randes ist im obigen Satz wesentlich. Dadurch wird gesichert, daß die Parallelflächen mindestens $C^{2}$-glatt sind, was die glättende Eigenschaft des Operators $K_{\partial D_{h}}$ sichert und somit die Gültigkeit der klassischen Lösungstheorie garantiert. Auch der Nachweis der Kompaktheit des Operators $K_{\partial D_{h}}$ als Abbildung von $C^{1, \alpha}\left(\partial D_{h}\right)$ nach $C^{1, \alpha}\left(\partial D_{h}\right)$, welcher die Abschwächung der $C^{3}$ Voraussetzung wohl ermöglichen würde (vgl. [Hähner2], S. 24), kann sich nicht auf die Direktwertdarstellungen des Gradientes des Doppelschichtpotentials (vgl. Satz 2.23 in [Colton/Kress]) stützen: weder existieren sie in der Literatur, noch ist uns deren Herleitung gelungen.

Für die Existenz von Lösungen bei $L^{2}$-Randdaten und für die Gültigkeit der im letzen Abschnitt beschriebenen Ansätze sorgt das folgende Lemma.

\section{Lemma 2.12}

Die Operatoren

$$
-\frac{1}{2} I_{3}+K_{3,(\partial D)}-S_{3,(\partial D)}:\left(L^{2}\right)^{3}(\partial D) \rightarrow\left(L^{2}\right)^{3}(\partial D)
$$

und

$$
-\frac{1}{2} I_{2}+K_{2,(\partial D)}+L-\frac{|\partial D|}{2 \pi} M:\left(L^{2}\right)^{2}(\partial D) \rightarrow\left(L^{2}\right)^{2}(\partial D)
$$

sind injektiv und beschränkt invertierbar.

Beweis: Man betrachte den dreidimensionalen Fall. Da die Operatoren $K_{3,(\partial D)}-$ $S_{3,(\partial D)}$ und $K_{2,(\partial D)}+L-\alpha$ in $L^{2}$ kompakt sind, reicht es, aus $\left(-\frac{1}{2} I_{3}+K_{3,(\partial D)}-\right.$ $\left.S_{3,(\partial D)}\right) \varphi=0$ bzw. $\left(-\frac{1}{2} I_{2}+K_{2,(\partial D)}+L-\alpha \varphi\right)$ mit $\varphi \in\left(L^{2}\right)^{3}$ bzw. $\varphi \in\left(L^{2}\right)^{2}$ auf $\varphi=0 \mathrm{zu}$ schließen. 
Mit dem bezüglich des Dualsystems $\left\langle L^{2}(\partial D), C(\partial D)\right\rangle$ mit $\langle f, g\rangle=\int_{\partial D} f g d s$ $\mathrm{zu}-\frac{1}{2} I_{3}+K_{3,(\partial D)}-S_{3,(\partial D)}$ adjungierten Operator $-\frac{1}{2} I_{3}+K_{3,(\partial D)}^{\prime}-S_{3,(\partial D)}$ liefert die Fredholmtheorie und schließlich die eindeutige Lösbarkeit der Integralgleichung 2.33) (Satz 2.9):

$$
\begin{array}{r}
\operatorname{dim} N\left(\left.\left(-\frac{1}{2} I_{3}+K_{3,(\partial D)}-S_{3,(\partial D)}\right)\right|_{L_{2}(\partial D)}\right)=\operatorname{dim} N\left(\left.\left(-\frac{1}{2} I_{3}+K_{3,(\partial D)}^{\prime}-S_{3,(\partial D)}\right)\right|_{C(\partial D)}\right) \\
=\operatorname{dim} N\left(\left.\left(-\frac{1}{2} I_{3}+K_{3,(\partial D)}-S_{3,(\partial D)}\right)\right|_{C(\partial D)}\right)=0 .
\end{array}
$$

Auf dieselbe Weise behandelt man den zweidimensionalen Fall. Die im Beweis von diesem Lemma verwendete Idee, zwei verschiedene Dualsysteme zu benutzen, geht auf Hähner ([Hähner2]) zurück. Der direkte Beweis der Aussage des Satzes ist auch möglich (vgl. Varnhorn]). 


\subsection{Numerische Behandlung des $\ddot{A} D P$ im $\mathbb{R}^{2}$}

Zur numerischen Prüfung der Rekonstruktionsgüte des inversen Algorithmus benötigen wir synthetische Daten. Um diese zu gewinnen, müssen wir das äußere Dirichlet Problem approximativ lösen können.

Wir geben hier der Vollständigkeit halber eine kurze Beschreibung des von uns zur Lösung der Integralgleichung 2.29 verwendeten Nyström Verfahrens, wobei auf eine detailliertere Darstellung in Evans verwiesen wird. Eine allgemeine Beschreibung des Nyström Verfahrens für Integralgleichungen bei stetigen bzw. schwach singulären Integralkernen sowie weiterführende Literaturangaben findet man in [Kress1. Da unser Zugang zum inversen Problem nicht auf wiederholtem Rechnen der direkten Probleme basiert, wird hier auch auf die Untersuchung der Konvergenz des Vorwärtslösers verzichtet; diese kann aber auf üblichem Weg geführt werden (vgl. Kress1, Kapitel 12).

\subsubsection{Das Nyström-Verfahren}

Für den Gebietsrand $\partial D$ sei die Existenz einer $2 \pi$-periodischen zweimal stetig differenzierbaren regulären $\left(z^{\prime}(t) \neq 0, \forall t \in[0,2 \pi]\right)$ Parametrisierung

$$
\partial D=\{z(t), 0 \leq t \leq 2 \pi\}
$$

angenommen. Das unmittelbare Einsetzen der Parametrisierung in die Kerne des Einfach- und Doppelschichtpotentials liefert

$A(t, \tau):=2 \Psi^{(r, c)}(z(t)-z(\tau))=\frac{\left|z^{\prime}(\tau)\right|}{2 \pi}\left(\ln \frac{1}{r(t, \tau)} \cdot I+\frac{(z(t)-z(\tau))(z(t)-z(\tau))^{T}}{r(t, \tau)^{2}}\right)$

und

$$
\begin{aligned}
B(t, \tau) & :=2 k_{2}^{(r)}(z(t), z(\tau)) \\
& =\frac{2}{\pi}\left((z(t)-z(\tau)) \cdot\left(z_{2}^{\prime}(\tau),-z_{1}^{\prime}(\tau)\right) \cdot \frac{(z(t)-z(\tau))(z(t)-z(\tau))^{T}}{r(t, \tau)^{4}}\right)
\end{aligned}
$$

mit $r(t, \tau):=|z(t)-z(\tau)|$ für alle $t \neq \tau$.

Während die Singularität in $B$ bei $t=\tau$ hebbar ist, erfordert die Integration der logarithmischen Singularität im Kern $A$ etwas Sorgfalt.

Wir stellen den singulären Term in der Form

$$
\ln \frac{1}{r(t, \tau)}=\ln \frac{2\left|\sin \frac{t-\tau}{2}\right|}{r(t, \tau)}-\frac{1}{2} \ln \left(4 \sin ^{2} \frac{t-\tau}{2}\right)
$$

dar, wobei der Term $\ln \frac{2\left|\sin \frac{t-\tau}{2}\right|}{r(t, \tau)}$ keine Singularität aufweist. 
Insgesamt erhalten wir für den Kern des Einfachschichtpotentials

$$
\begin{aligned}
A(t, \tau) & =-\frac{\left|z^{\prime}(\tau)\right|}{4 \pi} \ln \left(4 \sin ^{2} \frac{t-\tau}{2}\right) \cdot I+\tilde{A}(t, \tau) \\
\operatorname{mit} \quad \tilde{A}(t, \tau) & :=\frac{\left|z^{\prime}(\tau)\right|}{2 \pi}\left(\ln \frac{2\left|\sin \frac{t-\tau}{2}\right|}{r(t, \tau)} \cdot I+\frac{(z(t)-z(\tau))(z(t)-z(\tau))^{T}}{r(t, \tau)^{2}}\right) .
\end{aligned}
$$

Für $\tau \rightarrow t$ erhält man für $A$ und $B$

$$
\begin{aligned}
\tilde{A}(t, t) & =\frac{\left|z^{\prime}(t)\right|}{2 \pi}\left(\ln \frac{1}{\left|z^{\prime}(t)\right|} \cdot I+\frac{z^{\prime}(t) \cdot z^{\prime}(t)^{T}}{\left|z^{\prime}(t)\right|^{2}}\right) \\
\text { und } B(t, t) & =\frac{1}{\pi}\left(z_{2}^{\prime}(t) z_{1}^{\prime \prime}(t)-z_{1}^{\prime}(t) z_{2}^{\prime \prime}(t)\right) \cdot \frac{z^{\prime}(t) \cdot z^{\prime}(t)^{T}}{\left|z^{\prime}(t)\right|^{4}} .
\end{aligned}
$$

Setzt man jetzt

$$
g(t):=-2\left(f\left(z(t)+\frac{4 \pi}{\int_{0}^{2 \pi}\left|z^{\prime}(s)\right| d s} \int_{0}^{2 \pi} A(t, \tau) a_{\infty} d \tau\right)\right.
$$

so nimmt die Integralgleichung 2.29 nach der Parametrisierung die folgende Gestalt an:

$$
\begin{array}{r}
g(t)=\psi(t)+\int_{0}^{2 \pi}\{B(t, \tau)+ \\
\left.\eta\left(A(t, \tau)-\frac{\left|z^{\prime}(\tau)\right|}{\int_{0}^{2 \pi}\left|z^{\prime}(s)\right| d s} \int_{0}^{2 \pi} A(t, s) d s\right)+\frac{\eta \alpha}{2 \pi}\left|z^{\prime}(\tau)\right| I\right\} \cdot \psi(\tau) d \tau .
\end{array}
$$

Zur numerischen Approximation der hier vorkommenden Integrale wählen wir auf $[0,2 \pi)$ äquidistante Stützstellen $t_{j}:=\frac{\pi j}{m}, \quad j=0, \ldots, 2 m-1$ und benutzen die Quadraturformeln

$$
\int_{0}^{2 \pi} \ln \left(4 \sin ^{2} \frac{t-\tau}{2}\right) f(\tau) d \tau \approx \sum_{j=0}^{2 m-1} R_{j}^{(m)}(t) f\left(t_{j}\right) \quad 0 \leq t \leq 2 \pi
$$

mit den Gewichten

$$
R_{j}^{(m)}(t):=-\frac{2 \pi}{m} \sum_{k=0}^{m-1} \frac{1}{k} \cos k\left(t-t_{j}\right)-\frac{\pi}{m^{2}} \cos m\left(t-t_{j}\right) \quad \text { für } \quad j=0, \ldots, 2 m-1
$$

und die zusammengesetzte Trapezregel

$$
\int_{0}^{2 \pi} f(\tau) d \tau \approx \frac{\pi}{m} \sum_{j=0}^{2 m-1} f\left(t_{j}\right)
$$


Die Nyström Approximation zu 2.41 lautet

$$
\begin{aligned}
\psi^{(m)}(t)+\sum_{j=0}^{2 m-1}\left\{\frac { \pi } { m } \left[\left(t, t_{j}\right)+\eta(\right.\right. & \left.\tilde{A}\left(t, t_{j}\right)-\frac{\left|z^{\prime}\left(t_{j}\right)\right|}{D^{(m)}} \sum_{k=0}^{2 m-1}\left(\frac{\pi}{m} \tilde{A}\left(t, t_{k}\right)-R_{k}^{(m)}(t) \frac{\left|z^{\prime}\left(t_{k}\right)\right|}{4 \pi} \cdot I\right)\right) \\
& \left.\left.+\frac{\eta \alpha}{2 \pi}\left|z^{\prime}\left(t_{j}\right)\right| \cdot I\right]-\eta R_{j}^{(m)}(t) \frac{\left|z^{\prime}\left(t_{j}\right)\right|}{4 \pi} \cdot I\right\} \psi^{(m)}\left(t_{j}\right)=g(t),
\end{aligned}
$$

wobei

$$
D^{(m)}:=\frac{\pi}{m} \sum_{k=0}^{2 m-1}\left|z^{\prime}\left(t_{k}\right)\right|
$$

gesetzt ist.

Die vollständige Diskretisierung bezüglich $t$ an den $2 m$ Stützstellen führt nun mit $\psi_{i}^{(m)}:=\psi^{(m)}\left(t_{i}\right)$ für $i=0, \ldots, 2 m-1$ zum folgenden linearen Gleichungssystem:

$$
\begin{array}{r}
\psi_{i}^{(m)}+\sum_{j=0}^{2 m-1}\left\{\frac { \pi } { m } \left[B\left(t_{i}, t_{j}\right)+\eta\left(\tilde{A}\left(t_{i}, t_{j}\right)-\frac{\left|z^{\prime}\left(t_{j}\right)\right|}{D^{(m)}} \sum_{k=0}^{2 m-1}\left(\frac{\pi}{m} \tilde{A}\left(t_{i}, t_{k}\right)-R_{|i-k|}^{(m)} \frac{\left|z^{\prime}\left(t_{k}\right)\right|}{4 \pi} \cdot I\right)\right)\right.\right. \\
\left.\left.+\frac{\eta \alpha}{2 \pi}\left|z^{\prime}\left(t_{j}\right)\right| \cdot I\right]-\eta R_{|i-j|}^{(m)} \frac{\left|z^{\prime}\left(t_{j}\right)\right|}{4 \pi} \cdot I\right\} \psi_{j}^{(m)}=g\left(t_{i}\right)
\end{array}
$$

mit

$$
R_{j}^{(m)}:=R_{j}^{(m)}(0)=-\frac{2 \pi}{m} \sum_{k=1}^{m-1} \frac{1}{k} \cos \frac{k j \pi}{m}-\frac{(-1)^{j} \pi}{m^{2}} \quad \text { für } \quad j=0, \ldots, 2 m-1 .
$$

Dieses lineare Gleichungssystem besteht aus $4 m$ Gleichungen mit $4 m$ Unbekannten, da $\psi$ und $g$ zweidimensionale Vektorfelder darstellen. Setzen wir nun $\psi_{1 i}^{(m)}:=\psi_{1}^{(m)}\left(t_{i}\right)$ und $\psi_{2 i}^{(m)}:=\psi_{2}^{(m)}\left(t_{i}\right)$, so ergeben sich zwei Systeme

$$
\begin{aligned}
\psi_{1 i}^{(m)} & +\sum_{j=0}^{2 m-1}\left\{\frac { \pi } { m } \left[B_{11}\left(t_{i}, t_{j}\right)+\eta\left(\tilde{A}_{11}\left(t_{i}, t_{j}\right)-\frac{\left|z^{\prime}\left(t_{j}\right)\right|}{D^{(m)}} \sum_{k=0}^{2 m-1}\left(\frac{\pi}{m} \tilde{A}_{11}\left(t_{i}, t_{k}\right)-R_{|i-k|}^{(m)} \frac{\left|z^{\prime}\left(t_{k}\right)\right|}{4 \pi}\right)\right)\right.\right. \\
& \left.\left.+\frac{\eta \alpha}{2 \pi}\left|z^{\prime}\left(t_{j}\right)\right|\right]-\eta R_{|i-j|}^{(m)} \frac{\left|z^{\prime}\left(t_{j}\right)\right|}{4 \pi}\right\} \psi_{1 j}^{(m)} \\
& +\frac{\pi}{m} \sum_{j=0}^{2 m-1}\left\{B_{12}\left(t_{i}, t_{j}\right)+\eta\left(\tilde{A}_{12}\left(t_{i}, t_{j}\right)-\frac{\pi\left|z^{\prime}\left(t_{j}\right)\right|}{m D^{(m)}} \sum_{k=0}^{2 m-1} \tilde{A}_{12}\left(t_{i}, t_{k}\right)\right)\right\} \psi_{2 j}^{(m)} \\
& =g_{1}\left(t_{i}\right)
\end{aligned}
$$


und

$$
\begin{aligned}
\psi_{2 i}^{(m)} & +\frac{\pi}{m} \sum_{j=0}^{2 m-1}\left\{B_{21}\left(t_{i}, t_{j}\right)+\eta\left(\tilde{A}_{21}\left(t_{i}, t_{j}\right)-\frac{\pi\left|z^{\prime}\left(t_{j}\right)\right|}{m D^{(m)}} \sum_{k=0}^{2 m-1} \tilde{A}_{21}\left(t_{i}, t_{k}\right)\right)\right\} \psi_{1 j}^{(m)} \\
& +\sum_{j=0}^{2 m-1}\left\{\frac { \pi } { m } \left[B_{22}\left(t_{i}, t_{j}\right)+\eta\left(\tilde{A}_{22}\left(t_{i}, t_{j}\right)-\frac{\left|z^{\prime}\left(t_{j}\right)\right|}{D^{(m)}} \sum_{k=0}^{2 m-1}\left(\frac{\pi}{m} \tilde{A}_{22}\left(t_{i}, t_{k}\right)-R_{|i-k|}^{(m)} \frac{\left|z^{\prime}\left(t_{k}\right)\right|}{4 \pi}\right)\right)\right.\right. \\
& \left.\left.+\frac{\eta \alpha}{2 \pi}\left|z^{\prime}\left(t_{j}\right)\right|\right]-\eta R_{|i-j|}^{(m)} \frac{\left|z^{\prime}\left(t_{j}\right)\right|}{4 \pi}\right\} \psi_{2 j}^{(m)} \\
& =g_{2}\left(t_{i}\right)
\end{aligned}
$$

für $i=0, \ldots, 2 m-1$.

Nach Lösung dieser linearen Gleichungssysteme kennen wir also für $\psi_{1}^{(m)}$ und $\psi_{2}^{(m)}$ jeweils $2 m$ Werte.

$\mathrm{Um} u^{(m)} \mathrm{zu}$ berechnen, parametrisieren wir 2.31 und diskretisieren wieder an den $2 m$ Punkten $t_{j}:=\frac{\pi j}{m}, \quad j=0, \ldots, 2 m-1$.

Damit ergibt sich die Näherung an $u$ folgendermaßen:

$$
\begin{aligned}
& u^{(m)}(x)= \\
& -\frac{\pi}{m D^{(m)}} \sum_{j=0}^{2 m-1}\left|z^{\prime}\left(t_{j}\right)\right|\left(\ln \frac{1}{\left|x-z\left(t_{j}\right)\right|} \cdot I+\frac{\left(x-z\left(t_{j}\right)\right)\left(x-z\left(t_{j}\right)\right)^{T}}{\left|x-z\left(t_{j}\right)\right|^{2}}\right) a_{\infty} \\
& \quad-\frac{1}{m} \sum_{j=0}^{2 m-1}\left(\left(z_{2}^{\prime}\left(t_{j}\right)\left(x_{1}-z_{1}\left(t_{j}\right)\right)-z_{1}^{\prime}\left(t_{j}\right)\left(x_{2}-z_{2}\left(t_{j}\right)\right)\right) \frac{\left(x-z\left(t_{j}\right)\right)\left(x-z\left(t_{j}\right)\right)^{T}}{\left|x-z\left(t_{j}\right)\right|^{4}}\right) \psi_{j}^{(m)} \\
& +\frac{\eta}{4 m} \sum_{j=0}^{2 m-1}\left|z^{\prime}\left(t_{j}\right)\right|\left(\ln \frac{1}{\left|x-z\left(t_{j}\right)\right|} \cdot I+\frac{\left(x-z\left(t_{j}\right)\right)\left(x-z\left(t_{j}\right)\right)^{T}}{\left|x-z\left(t_{j}\right)\right|^{2}}\right) \psi_{j}^{(m)} \\
& +\frac{\eta \pi}{4 m^{2} D^{(m)}} \sum_{j=0}^{2 m-1}\left\{\left|z^{\prime}\left(t_{j}\right)\right|\left(\ln \frac{1}{\left|x-z\left(t_{j}\right)\right|} \cdot I+\frac{\left(x-z\left(t_{j}\right)\right)\left(x-z\left(t_{j}\right)\right)^{T}}{\left|x-z\left(t_{j}\right)\right|^{2}}\right) \sum_{k=0}^{2 m-1}\left|z^{\prime}\left(t_{k}\right)\right| \psi_{k}^{(m)}\right\} \\
& \quad-\frac{\eta \alpha}{4 m} \sum_{j=0}^{2 m-1}\left|z^{\prime}\left(t_{j}\right)\right| \psi_{j}
\end{aligned}
$$

für alle $x \in D^{+}$.

\subsubsection{Numerische Beispiele}

Einige numerischen Beispiele sollen nun die Qualität des im vorhergehenden Abschnitt beschriebenen Verfahrens illustrieren. 


\section{Beispiel 2.1}

Als Gebiet für den ersten Test wurde die Ellipse $\mathbf{E}$ mit dem Rand

$$
\partial \mathbf{E}=\{x(t)=(2 \cos t, \sin t), t \in[0,2 \pi]\}
$$

gewählt. Als Randbedingung auf $\partial \mathbf{E}$ geben wir die Werte des im Punkt $(0.3,0)$ (also innerhalb der Ellipse) plazierten Stokeslets der Stärke $(1,2)$ vor.

Das Verfahren wurde in den Punkten $x_{1}=(-5,10)$ und $x_{2}=\left(5 * 10^{8}, 5 * 10^{8}\right)$ bei $\alpha=1, \eta=2$ getestet, wobei folgende Ergebnisse erzielt wurden:

\begin{tabular}{||l|l|l|l|l||}
\hline$n$ & $A F_{n}\left[u_{1}\left(x_{1}\right)\right]$ & $A F_{n}\left[u_{2}\left(x_{1}\right)\right]$ & $A F_{n}\left[u_{1}\left(x_{2}\right)\right]$ & $A F_{n}\left[u_{2}\left(x_{2}\right)\right]$ \\
\hline 4 & $8.5349870379 \mathrm{e}-03$ & $1.6893113158 \mathrm{e}-02$ & $3.4851970802 \mathrm{e}-03$ & $8.4889935558 \mathrm{e}-03$ \\
8 & $1.8792408390 \mathrm{e}-04$ & $3.6889977810 \mathrm{e}-04$ & $1.6636721972 \mathrm{e}-04$ & $4.0431221250 \mathrm{e}-04$ \\
16 & $2.0070175299 \mathrm{e}-08$ & $3.6349095733 \mathrm{e}-08$ & $2.0559205893 \mathrm{e}-08$ & $4.5513132818 \mathrm{e}-08$ \\
32 & $1.3322676296 \mathrm{e}-15$ & $1.3322676296 \mathrm{e}-15$ & $1.0658141036 \mathrm{e}-14$ & $2.1316282073 \mathrm{e}-14$ \\
64 & $2.2204460493 \mathrm{e}-15$ & $2.2204460493 \mathrm{e}-15$ & $3.5527136788 \mathrm{e}-15$ & $1.4210854715 \mathrm{e}-14$ \\
\hline
\end{tabular}

Tabelle 2.1: Ergebnisse zu Beispiel 2.1

Mit $A F_{n}\left[u_{i}\left(x_{j}\right)\right], i, j=1,2$ ist hier der absolute Fehler in der i-ten Komponente der Lösung im Punkt $x_{j}$ bezeichet; $n$ steht für die Zahl der für das Verfahren gewählten Stützpunkte.

Dieses Beispiel demonstriert eine mindestens exponentielle, schnell eingreifende Konvergenz der numerischen Methode. Im Falle analytischer rechter Seiten des zu lösenden Integralgleichungssystems und bei einer analytischen Parametrisierung des Gebietsrandes ist zu erwarten, daß auch die Lösung der Gleichung analytisch ist. Da die Konvergenzstärke des Nyströmverfahrens von der Güte der Quadraturformeln direkt abhängig ist (und bei der trigonometrischen Interpolation analytischer periodischer Funktionen verschwindet der Fehler exponentiell, S.[Kress1], Satz 11.7), muß man mit einem exponentiellen Abklingen des Fehlers des numerischen Ergebnisses rechnen.

Die Änderung der Kopplungsparameter bei der Ellipse verändert die Konvergenzgeschwindigkeit kaum:

\begin{tabular}{||l|l|l||l|l||}
\hline$n$ & $A F_{n}\left[u_{1}\left(x_{1}\right)\right]$ & $A F_{n}\left[u_{2}\left(x_{1}\right)\right]$ & $A F_{n}\left[u_{2}\left(x_{1}\right)\right]$ & $A F_{n}\left[u_{1}\left(x_{2}\right)\right]$ \\
\hline 4 & $1.1473597236 \mathrm{e}-02$ & $3.2888513862 \mathrm{e}-01$ & $1.8265892017 \mathrm{e}-06$ & $3.0560895681 \mathrm{e}-06$ \\
8 & $1.2719126993 \mathrm{e}-04$ & $1.2613809834 \mathrm{e}-03$ & $1.0316357079 \mathrm{e}-04$ & $1.2373954937 \mathrm{e}-03$ \\
16 & $2.0070032303 \mathrm{e}-08$ & $3.6346330834 \mathrm{e}-08$ & $2.0559205893 \mathrm{e}-08$ & $4.5513083080 \mathrm{e}-08$ \\
32 & $3.9968028887 \mathrm{e}-15$ & $2.0872192863 \mathrm{e}-14$ & $1.0658141036 \mathrm{e}-14$ & $4.2632564146 \mathrm{e}-14$ \\
64 & $0.0000000000 \mathrm{e}+00$ & $1.8207657604 \mathrm{e}-14$ & $0.0000000000 \mathrm{e}+00$ & $2.1316282073 \mathrm{e}-14$ \\
\hline
\end{tabular}

Tabelle 2.2: Beispiel 2.1 mit $\eta=\alpha=0.001$ 


\section{Beispiel 2.2}

Nun lösen wir das ÄDP auf dem Gebiet D der Gestalt

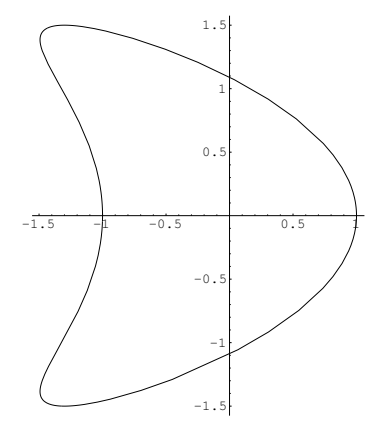

Abbildung 2.1: Das Gebiet D

mit dem Rand

$$
\partial \mathbf{D}=x(t)=(\cos t+0.65 \cos (2 t)-0.65,1.5 \sin t), t \in[0,2 \pi] .
$$

Die sonstigen Parameter wurden aus dem ersten Beispiel übernommen.

Aus den nachstehenden Tabellen wird wieder das exponentielle Konvergenzverhalten des Nyströmverfahrens ersichtlich; im Vergleich zur Ellipse klingt der Approximationsfehler bei wachsendem $n$ jedoch langsamer ab. Deutlicher zeigt sich auch die Abhängigkeit der Ergebnisqualität von der Wahl der Kopplungsparameter.

\begin{tabular}{||l|l|l|l|l||}
\hline$n$ & $A F_{n}\left[u_{1}\left(x_{1}\right)\right]$ & $A F_{n}\left[u_{2}\left(x_{1}\right)\right]$ & $A F_{n}\left[u_{1}\left(x_{2}\right)\right]$ & $A F_{n}\left[u_{2}\left(x_{2}\right)\right]$ \\
\hline 4 & $3.3320525646 \mathrm{e}-02$ & $6.0632272294 \mathrm{e}-02$ & $3.0991555853 \mathrm{e}-02$ & $7.4792271141 \mathrm{e}-02$ \\
8 & $2.7166147022 \mathrm{e}-03$ & $9.9733810727 \mathrm{e}-03$ & $1.8922424503 \mathrm{e}-03$ & $8.6767506788 \mathrm{e}-03$ \\
16 & $1.6778921381 \mathrm{e}-06$ & $6.7832735215 \mathrm{e}-05$ & $5.3497321062 \mathrm{e}-07$ & $5.5733044682 \mathrm{e}-05$ \\
32 & $6.0930815948 \mathrm{e}-10$ & $7.6532824522 \mathrm{e}-10$ & $4.5701042950 \mathrm{e}-10$ & $5.6071769450 \mathrm{e}-10$ \\
64 & $0.0000000000 \mathrm{e}+00$ & $1.3322676296 \mathrm{e}-15$ & $1.0658141036 \mathrm{e}-14$ & $1.4210854715 \mathrm{e}-14$ \\
\hline
\end{tabular}

Tabelle 2.3: Ergebnisse zu Beispiel 2.2 bei $\eta=2, \alpha=1$

\begin{tabular}{||l|l|l|l|l||}
\hline$n$ & $A F_{n}\left[u_{1}\left(x_{1}\right)\right]$ & $A F_{n}\left[u_{2}\left(x_{1}\right)\right]$ & $A F_{n}\left[u_{1}\left(x_{2}\right)\right]$ & $A F_{n}\left[u_{2}\left(x_{2}\right)\right]$ \\
\hline 4 & $1.4608389637 \mathrm{e}-01$ & $1.8621094486 \mathrm{e}-03$ & $4.5398407487 \mathrm{e}-06$ & $6.5748373146 \mathrm{e}-07$ \\
8 & $2.3403585925 \mathrm{e}-01$ & $1.6834774459 \mathrm{e}-01$ & $1.4571635301 \mathrm{e}-05$ & $2.2723967857 \mathrm{e}-04$ \\
16 & $9.4359681154 \mathrm{e}-03$ & $5.9161722190 \mathrm{e}-03$ & $2.3278338759 \mathrm{e}-06$ & $9.6597003075 \mathrm{e}-04$ \\
32 & $4.2732144045 \mathrm{e}-07$ & $1.3979335600 \mathrm{e}-07$ & $3.4730192056 \mathrm{e}-09$ & $3.7509266804 \mathrm{e}-07$ \\
64 & $1.2878587086 \mathrm{e}-14$ & $2.6645352591 \mathrm{e}-15$ & $3.9079850467 \mathrm{e}-14$ & $1.4210854715 \mathrm{e}-14$ \\
\hline
\end{tabular}

Tabelle 2.4: Ergebnisse zu Beispiel 2.2 bei $\eta=\alpha=0.001$ 


\section{Kapitel 3}

\section{Inverses Problem}

Seien $D$ und $B$ beschränkte $C^{3}$-glatte Gebiete ohne Löcher in $\mathbb{R}^{m}$ mit $m=2,3$; das Gebiet $D$ sei samt Rand in $B$ vollständig enthalten mit $\partial D \cap \partial B=\emptyset$.

Unter dem inversen Problem verstehen wir die Aufgabe der Rekonstruktion des Gebiets $D$ aus der Kenntnis von Lösungen $u_{i}(x, z), i=1, \ldots, m$ der äußeren Dirichlet Probleme zu der Stokes-Gleichung mit den Randbedingungen

$$
u_{i}=-\Psi_{m}^{(r, c)}(\cdot-z) \cdot e_{i} \quad \text { auf } \partial D
$$

für alle $x, z \in \partial B$, wobei mit $e_{i}, i=1, \ldots, m$ die Einheitsvektoren in $\mathbb{R}^{m}$ bezeichnet sind.

Im zweidimensionalen Fall gehen wir davon aus, daß die Lösungen im Unendlichen beschränkt und deren asymptotischen Werte $u_{i, \infty}(z)$ bekannt sind.

Die Linearität der Stokes-Gleichung erlaubt es, die Ausgangsinformation für das inverse Problem auf folgende Weise zusammenzufassen :

Für alle $x \in \partial B$ ist die durch $z \in \partial B$ parametrisierte Familie von Funktionalmatrizen $w(x, z)$ bekannt mit der Eigenschaft: Die Funktionen $w(x, z) \cdot a$ sind Geschwindigkeitskomponenten von beschränkten Lösungen des ÄDP zu den Randdaten $-\Psi_{m}^{(r, c)}(\cdot, z) \cdot a, a \in \mathbb{R}^{m}$.

Im zweidimensionalen Fall sind auch die Werte der Lösungen im Unendlichen, also die Matrix $w_{\infty}(z)$, bekannt.

Eine zentrale Rolle in diesem Kapitel spielt der Operator $F_{m}: L^{2}(\partial B) \rightarrow L^{2}(\partial B)$, gegeben für $m=3$ durch

$$
\left(F_{3} g\right)(x):=-\int_{\partial B} w(x, y) g(y) d s(y) \quad x \in \partial B
$$


und für $m=2$ durch

$$
\left(F_{2} g\right)(x):=-\int_{\partial B}\left[w(x, y)-w_{\infty}(y)\right] g(y) d s(y), \quad x \in \partial B .
$$

Unsere Aufgabe ist zu zeigen, wie die Kenntnis der Eigenwerte und der Eigenvektoren des „Meßoperators“ $F_{m}$ eine Charakterisierung des Gebietes $D$ ermöglicht.

\subsection{Der dreidimensionale Fall}

\subsubsection{Faktorisierung des Meßoperators}

Wir untersuchen als erstes den Operator $G:\left.f \rightarrow u\right|_{\partial B}$, welcher die auf $\partial D$ gegebenen Randdaten $f$ in die $\partial B$ Spur der Lösung $u$ des ÄDP mit diesen Randdaten abbildet.Die eindeutige Lösbarkeit des ÄDP bei $L^{2}$-Randdaten und die Darstellung 2.32 der Lösung ermöglichen uns den Beweis folgender Aussage:

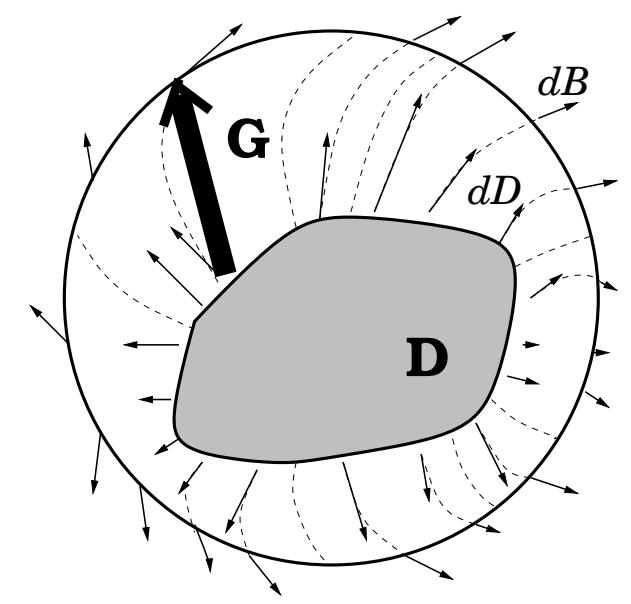

Satz 3.1

Der Operator $G: L^{2}(\partial D) \rightarrow L^{2}(\partial B)$ ist kompakt und injektiv und sein adjungierter Operator $G^{*}: L^{2}(\partial B) \rightarrow L^{2}(\partial D)$ ebenfalls.

Beweis:

Für den Operator $G$ ergibt sich aus 2.32 und 2.33 die Darstellung

$$
G=U\left(-\frac{1}{2} I+K_{(\partial D)}-S_{(\partial D)}\right)^{-1}
$$

mit dem Operator

$$
(U \varphi)(x):=\int_{\partial D}\left(k_{3}^{(r)}(x, y)-\Psi^{(r, c)}(x, y)\right) \varphi(y) d s(y), \quad x \in \partial B .
$$

Der Kern des Integraloperators $U$ ist stetig, nach Lemma 2.12 ist die Injektivität und die Beschränktheit des Operators $\left(-\frac{1}{2} I+K_{(\partial D)}-S_{(\partial D)}\right)^{-1}: L^{2}(\partial D) \rightarrow L^{2}(\partial D)$ bereits bekannt. Daher ist $G$ kompakt.

Wir zeigen jetzt, daß aus $U \varphi=0$ mit einem $\varphi \in L^{2}(\partial D)$ stets $\varphi=0$ folgt. Dazu definiere man für $x \in D^{+}$die Funktion $u(x):=(K \varphi-S \varphi)(x)$. Gemäß der Voraussetzung gilt $\left.u\right|_{\partial B}=U \varphi=0$. Nun sorgt die Eindeutigkeit des ÄDP im gesamten 
Analyzititätbereich von $u$, d.h. in $D^{+}$, für $u=0$. Die $L^{2}$-Sprungbeziehungen liefern $-\frac{1}{2} \varphi+K_{(\partial D)} \varphi-S_{(\partial D)} \varphi=0$ auf $\partial D$ und die Injektivität von $\left(-\frac{1}{2} I+K_{(\partial D)}-S_{(\partial D)}\right)$ besagt $\varphi \equiv 0$.

Der Operator $G^{*}: L^{2}(\partial B) \rightarrow L^{2}(\partial D)$ ist als

$$
G^{*}=\left(-\frac{1}{2} I+K_{(\partial D)}^{\prime}-S_{(\partial D)}\right)^{-1} U^{*}
$$

mit

$$
U^{*} \varphi=\left.\left[\left(\tilde{K}^{\prime}-\tilde{S}\right) \varphi\right]\right|_{\partial D}
$$

darstellbar, wobei mit $\tilde{S}$ und $\tilde{K}^{\prime}$ das Einfachschichtpotential einer auf $\partial B$ definierten Dichte sowie seine Normalspannung bezeichnet sind.

Da der Operator $P:=\left(-\frac{1}{2} I+\tilde{K}_{(\partial B)}^{\prime}-\tilde{S}_{(\partial B)}\right)^{-1}$ injektiv ist (wegen 2.12 mit $\partial B$ statt $\partial D$ und der Fredholmtheorie), folgt die Injektivität von $G^{*}$ aus der von $U^{*}$; diese ist unschwer zu sehen: Die Gleichung $\left.\left[\left(\tilde{K}^{\prime}-\tilde{S}\right) \varphi\right]\right|_{\partial D}=0$ impliziert, wegen der eindeutigen Lösbarkeit des inneren sowie des äußeren Dirichletproblems zu den homogenen Daten auf $\partial D$, daß $\left(\tilde{K}^{\prime}-\tilde{S}\right) \varphi=0$ überall in $B$ gilt. Die Sprungbeziehungen für $\tilde{S}$ und $\tilde{K}^{\prime}$ sowie wiederum die Injektivität von $P$ liefern nun die Injektivität von $U^{*}$.

Der Zusammenhang zwischen den Operatoren $F_{3}$ und $G$ wird aus der folgenden faktorisierten Form von $F_{3}$ ersichtlich:

\section{Satz 3.2}

Für den Operator $F_{3}$ gilt die Darstellung

$$
F_{3}=G S G^{*}
$$

Beweis:

Mit der Definition von $G$ und der Randbedingung 3.1 folgt für alle $y \in \partial B$

$$
-\left.w(\cdot, y)\right|_{\partial B}=\left.G \Psi^{(r, c)}(\cdot-y)\right|_{\partial D}, \quad \forall y \in \partial B
$$

Man multipliziere dies mit einem $g \in L^{2}(\partial B)$ und integriere über $\partial B$. Aus der Eindeutigkeit des ÄDP und der Stetigkeit von $G$ folgert man

$$
F_{3} g=G \int_{\partial B} \Psi^{(r, c)}(x-y) g(y) d s(y), \quad x \in \partial B .
$$

Mit dem Hilfsoperator $A: L^{2}(\partial B) \rightarrow L^{2}(\partial D):$

$$
(A g)(x):=\int_{\partial B} \Psi^{(r, c)}(x-y) g(y) d s(y), \quad x \in \partial D
$$


schreiben wir 3.7 als

$$
F_{3}=G A .
$$

Andererseits kann der Adjungierte von $A, A^{*}: L^{2}(\partial D) \rightarrow L^{2}(\partial B)$ :

$$
\left(A^{*} \varphi\right)(x):=\int_{\partial D} \Psi^{(r, c)}(x-y) \varphi(y) d s(y), \quad x \in \partial B
$$

nach der Definition von $A, G$ und $S$ als $A^{*}=G S$ dargestellt werden. Es gilt folglich $A=S G^{*}$. Dies, kombiniert mit 3.8, ergibt die gewünschte Faktorisierung 3.6.

Die Faktorisierung 3.6 besagt mehr über die Eigenschaften des Operators $F_{3}$ als seine Integralform; der Operator $S_{(\partial D)}$ enthält alleine schon, und expliziter als $F_{3}$, die Information über die Fläche $\partial D$, denn das Äußere Dirichlet Problem mit der Randbedingung 3.1 ist auch mittels Einfachschichtpotentialansatz lösbar (vgl. das nachstehende Lemma). Für das Weitere fassen wir die Eigenschaften des Operators $S_{(\partial D)}$ zusammen.

\section{Lemma 3.3}

Der kompakte und selbsadjungierte Randintegraloperator $S_{(\partial D)}: L^{2}(\partial D) \rightarrow L^{2}(\partial D)$ ist positiv semi-definit. Sein Nullraum ist gegeben durch

$$
N\left(S_{(\partial D)}\right)=\operatorname{span}\left\{\nu_{\partial D}\right\}
$$

Als Operator von $H_{\perp \nu}^{-\frac{1}{2}}(\partial D)$ nach $H_{\perp \nu}^{\frac{1}{2}}(\partial D)$ ist $S_{(\partial D)}$ bijektiv.

Beweis:

Mit einem $\varphi \in C(\partial D)$ bilde man das vollständige Einfachschichtpotential $(u, p)=$ $\left(u_{\varphi}, p_{\varphi}\right):=\boldsymbol{S}_{3} \varphi$. Die Greensche Formel 2.3, angewandt auf $(u, p)$, ergibt

$$
\int_{\partial D} T_{p}^{u_{-}} \nu \cdot u_{-} d s=2 \int_{D} D u: D u d y
$$

in $D$ und

$$
\int_{\partial D} T_{p}^{u_{+}} \nu \cdot u_{+} d s=-2 \int_{D^{+}} D u: D u d y
$$

in $D^{+}$. Mit der Stetigkeit von $u$ (Satz 2.2) und der Sprungbeziehung 2.11 folgt also

$$
\int_{\partial D} \varphi S \varphi d s=2 \int_{\mathbb{R}^{3}}|D u|^{2} d y \geq 0 .
$$

Somit ist die Einschränkung von $S$ auf $C(\partial D)$ positiv semi-definit. Da aber der Raum $C(\partial D)$ in $L^{2}(\partial D)$ dicht ist und der Operator $S$ stetig, ist $S$ auch in $L^{2}(\partial D)$ positiv semi-definit. 
Im Falle $(\varphi, S \varphi)=0$ tritt in 3.8 mit einem $\varphi \in L^{2}(\partial D)$ die Gleichheit ein und die Deformationsmatrix $D u_{\varphi}$ verschwindet, was zusammen mit dem Abklingverhalten (2.23) des Einfachschichtpotentials das Verschwinden von $u$ zunächst in $D^{+}$, dann nach Stetigkeit von $u$ in $\overline{D^{+}}$und schließlich wegen der Eindeutigkeit des inneren Dirichlet-Problems (s. Satz 2.6) auch in $D$ sichert. Also ist $u=0$ in $\mathbb{R}^{3}$.

Die Stokes-Gleichung besagt jetzt grad $p=0$ in $\mathbb{R}^{3}$. In $D$ gilt also $p=$ const und in $D^{+}$, wegen 2.24, $p=0$.

Aus den Sprungbeziehungen 2.11 in ihrer $L^{2}$-Form schließen wir auf $\varphi=c \nu_{\partial D}$ mit einer Konstante $c$.

Den Beweis der letzten Aussage des Satzes findet man z.B. in [Dautray/Lions];

der Beweis der Stetigkeit des Operators $S^{-1}: H_{\perp \nu}^{\frac{1}{2}}(\partial D) \rightarrow H_{\perp \nu}^{-\frac{1}{2}}(\partial D)$ erfolgt mit Hilfe der Anwendung des Satzes vom Lax-Milgram auf die variationelle Form der Gleichung $S \varphi=f$.

\section{Satz 3.4}

Der Operator $F_{3}$ ist kompakt und positiv semi-definit. Sein Nullraum ist gegeben durch

$$
N\left(F_{3}\right)=\operatorname{span}\left\{\nu_{\partial B}\right\} .
$$

Beweis:

Aus 3.6 sieht man, daß $F_{3}$ kompakt und selbstadjungiert ist.

Der Operator $G$ ist nach Satz 3.1 injektiv und $S_{\partial D}$ ist nach Lemma 3.3 positiv semi-definit. Damit folgt aus

$$
\left(F_{3} g, g\right)=\left(S G^{*} g, G^{*} g\right),
$$

daß $F_{3}$ positiv-semidefinit ist mit

$$
N\left(F_{3}\right)=N\left(S G^{*}\right)=N(A) .
$$

Da der Operator $A$ eine Einschränkung des Einfachschichtpotentialoperators ist, folgt

$$
N(A)=\operatorname{span}\left\{\nu_{\partial B}\right\}
$$

nach dem Lemma 3.3. 


\subsubsection{Charakterisierung des Gebiets}

Die in dem Satz 3.4 festgestellten Eigenschaften des Operators $F_{3}$ erlauben die Anwendung des Satzes über das Spektrum selbstadjungierter kompakter Operatoren auf $F_{3}$. Somit erhalten wir, daß die orthonormierten Eigenfunktionen

$$
g_{j} \in L^{2}(\partial B), j \in \mathbb{N}
$$

des Operators $F_{3}$ eine vollständige orthonormale Basis in

$$
L_{\perp \nu}^{2}(\partial B):=\left\{\varphi \in L^{2}(\partial B):\left\langle\varphi,\left.\nu\right|_{\partial B}>_{L^{2}(\partial B)}=0\right\}\right.
$$

darstellen, wobei die entsprechenden Eigenwerte eine Folge von positiven reellen Zahlen

$$
\lambda_{1} \geq \lambda_{2} \geq \ldots>0 .
$$

bilden.

Da der Operator $S: L_{\perp \nu}^{2}(\partial D) \rightarrow L_{\perp \nu}^{2}(\partial D)$ kompakt und positiv definit ist, besitzt er eine ebenfalls kompakte und in $L_{\perp \nu}^{2}(\partial D)$ positiv definite Wurzel $S^{\frac{1}{2}}$; diese ist eindeutig definiert.

Mit dieser Definition seien die Funktionen

$$
\varphi_{n}:=\frac{1}{\sqrt{\lambda_{n}}} S^{\frac{1}{2}} G^{*} g_{n}, \quad n=1, \ldots
$$

deklariert.

\section{Lemma 3.5}

Die Funktionen $\varphi_{n}, n=1, \ldots$ bilden eine vollständige orthonormale Basis von $L_{\perp \nu}^{2}(\partial D)$.

Beweis:

Die Orthogonalität des Systems $\varphi_{n}, n=1, \ldots$ folgt aus der Orthogonalität von $g_{n}$ :

$$
\left(\varphi_{i}, \varphi_{j}\right)=\frac{1}{\sqrt{\lambda_{i} \lambda_{j}}}\left(S^{\frac{1}{2}} G^{*} g_{i}, S^{\frac{1}{2}} G^{*} g_{j}\right)=\frac{1}{\sqrt{\lambda_{i} \lambda_{j}}}<F g_{i}, g_{j}>=\delta_{i, j} .
$$

Für ein $\varphi \in L_{\perp \nu_{\partial D}}^{2}$ mit $\left(\varphi, \varphi_{n}\right)=0$ für alle $n=1, \ldots$ gilt

$$
0=\left(\varphi, \varphi_{i}\right)=\frac{1}{\sqrt{\lambda_{i}}}\left(\varphi, S^{\frac{1}{2}} G^{*} g_{i}\right)=\left(G S^{\frac{1}{2}} \varphi, g_{i}\right), \quad i=1,2,3 \ldots
$$

Die Vollständigkeit von $g_{n}$ erfordert also, daß die homogene Gleichung $G S^{\frac{1}{2}} \varphi=0$ erfüllt ist; wegen der Injektivität von $G S^{\frac{1}{2}}$ auf $L_{\perp \nu}^{2}$ folgt $\varphi=0$ und die Vollständigkeit von $\varphi_{n}, n=1, \ldots$ ist bewiesen.

Diese Basis erlaubt uns, mit Hilfe der Eigenfunktionen von $F_{3}$ den Wertebereich des Operators $G S^{\frac{1}{2}}$ zu beschreiben: 


\section{Lemma 3.6}

Eine Funktion $f \in L_{\perp \nu}^{2}(\partial B)$ liegt in $G S^{\frac{1}{2}}\left(L_{\perp \nu}^{2}(\partial D)\right)$ dann und nur dann, wenn die Reihe

$$
\sum_{i=1}^{\infty} \frac{\left|\left(f, g_{i}\right)\right|^{2}}{\lambda_{i}}
$$

konvergiert.

Beweis: Der folgende Beweis ist formal identisch mit dem des Lemmas 5 in [Hähner1] mit $L_{\perp \nu}^{2}$ hier statt $L^{2}$ dort.

Man schreibe ein $f=G S^{\frac{1}{2}} \varphi$ mit $\varphi \in L_{\perp \nu}^{2}(\partial D), \varphi=\sum_{i=1}^{\infty} \alpha_{i} \varphi_{i}$ als

$$
f=\sum_{i=1}^{\infty} \alpha_{i} G S^{\frac{1}{2}} \varphi_{i}
$$

Mit der Definition 3.12 von $\varphi_{i}$ und der Faktorisierung 3.6 des Operators $F_{3}$ erhält man

$$
f=\sum_{i=1}^{\infty} \frac{\alpha_{i}}{\sqrt{\lambda_{i}}} F_{3} g_{i}=\sum_{i=1}^{\infty} \alpha_{i} \sqrt{\lambda_{i}} g_{i}
$$

und somit gilt $\alpha_{i}=\frac{\left(f, g_{i}\right)}{\sqrt{\lambda_{i}}}$. Die Konvergenz der Reihe 3.13 ergibt sich aus der Parsevalschen Gleichung.

Umgekehrt, sei die Konvergenz von 3.13 vorausgesetzt. Für die Funktion

$$
\varphi:=\sum_{i=1}^{\infty} \frac{\left(f, g_{i}\right)}{\sqrt{\lambda_{i}}} \varphi_{i}=\sum_{i=1}^{\infty} \alpha_{i} \sqrt{\lambda_{i}} g_{i} \in L_{\perp \nu}^{2}(\partial D)
$$

erhalten wir mit 3.12 die Beziehung $G S^{\frac{1}{2}} \varphi=f$.

$\mathrm{Zu}$ jedem Punkt $z \in B$ definiere man nun eine Funktion $f_{z}(x):=\Psi^{(r, c)}(x-z) a$, $x \in \partial B$ mit einem $a=$ const $\in \mathbb{R}^{3}, a \neq 0$.

Diese Funktionen ermöglichen uns, für jeweiliges $z \in B$ anhand von Spektraldaten von $F_{3}$ die Entscheidung zu treffen, ob z in $D$ liegt oder nicht; dies zeigt der nächste Satz.

\section{Satz 3.7}

Für $z \in B$ sind die beiden folgenden Aussagen äquivalent:

1) $z \in D$

2) $\sum_{j=1}^{\infty} \frac{\left|\left(f_{z}, g_{j}\right)\right|^{2}}{\lambda_{j}}<\infty$ 


\section{Beweis:}

Sei zunächst $z \in D$. Die Funktion $\Psi^{(r, c)}(\cdot-z) a$ ist analytisch in $D^{+}$und verschwindet im Unendlichen. Darüber hinaus gilt $\int_{\partial D} \nu(x) \cdot \Psi^{(r, c)}(x-z) a d s(x)=0$, denn für eine ausreichend große Kugel $B_{R} \supset B$ mit Zentrum in z folgt mit dem Gaußschen Satz, mit der Divergenzfreiheit von $\Psi^{(r, c)}(\cdot-z) a$ in $B_{R} \backslash B$ und der Definition von $\Psi^{(r, c)}$

$$
\begin{aligned}
& \int_{\partial D} \nu(x) \cdot \Psi^{(r, c)}(x-z) a d s(x)=\int_{\partial B_{R}} \nu(x) \cdot \Psi^{(r, c)}(x-z) a d s(x) \\
& =c_{1} \int_{\partial B_{R}} \nu(x) \cdot \frac{1}{R} a d s(x)+c_{2} \int_{\partial B_{R}} \nu(x) \cdot \frac{1}{R^{3}} x x \cdot a d s(x) \\
& =c_{3} \int_{\partial B_{R}} \nu(x) \cdot a d s(x)=c_{3} \int_{B_{R}} \operatorname{div} a=0
\end{aligned}
$$

mit gewissen Konstanten $c_{1}, c_{2}, c_{3}$. Die Funktion $f_{z}$ liegt somit in $H_{\perp \nu}^{\frac{1}{2}}(\partial D)$.

Wegen der eindeutigen Lösbarkeit des ÄDP mit den Randdaten

$$
\left.\Psi^{(r, c)}(\cdot-z) a\right|_{\partial D} \in H_{\perp \nu}^{\frac{1}{2}}(\partial D)
$$

gilt $f=G\left(\left.\Psi^{(r, c)}(\cdot-z) a\right|_{\partial D}\right)$.

Nach Lemma 3.3 existiert genau eine Funktion $\varphi \in H_{\perp \nu}^{-\frac{1}{2}}(\partial D)$ mit

$$
\left.\Psi^{(r, c)}(\cdot-z) a\right|_{\partial D}=S \varphi=S^{\frac{1}{2}}\left(S^{\frac{1}{2}} \varphi\right) \in S^{\frac{1}{2}}\left(L_{\perp \nu}^{2}(\partial D)\right)
$$

Also ist

$$
f_{z}=\left.G \Psi^{(r, c)}(\cdot-z)\right|_{\partial D}=G S^{\frac{1}{2}}\left(S^{\frac{1}{2}} \varphi\right)
$$

und die Reihe 3.14 konvergiert nach Lemma 3.6 .

Sei jetzt $z \in \partial D$. Die Funktion $\Psi^{(r, c)}(\cdot-z) a$ ist die einzige, die in $D^{+}$analytisch ist, im Unendlichen verschwindet und auf $\partial B$ mit $f_{z}$ zusammenfällt. Wegen der Singularität von $\Psi^{(r, c)} a$ im Punkt $z$ gilt aber $\left.\Psi^{(r, c)}\right|_{\partial D} \notin L^{2}(\partial D)$, somit

$$
\left.\Psi^{(r, c)}\right|_{\partial D} \notin S^{\frac{1}{2}}\left(L^{2}(\partial D)\right) \subset L^{2}(\partial D) .
$$

Demzufolge gilt $f_{z} \notin G S^{\frac{1}{2}}\left(L^{2}(\partial D)\right)$ und die Reihe 3.14 divergiert.

Auf analoge Weise stellt man auch im Falle $z \in D^{+} \cap B$ fest, daß3.14 nicht konvergieren kann. 


\subsection{Der zweidimensionale Fall}

Die im letzten Abschnitt beschriebene Vorgehensweise zur Behandlung des inversen Problems soll auf den zweidimensionalen Fall übertragen werden. Da die Lösungen des $\ddot{A D P}$ in $\mathbb{R}^{2}$ im Unendlichen i. A. nicht abklingen, ist die Greensche Formel für unbeschränkte Gebiete nicht immer anwendbar. Dadurch kann insbesondere der Beweis der positiven Definitheit des Einfachschichtpotentialoperators nicht für alle Dichten wie im räumlichen Fall (vgl. Lemma 3.3) geführt werden.

Bei dem analogen inversen Problem zu der Laplace Gleichung, wo ein ähnliches Problem auftritt, wurde diese Schwierigkeit in [Kress2] elegant umgangen; wir zeigen, daß die dort vorgeschlagenen Modifikationen auch im hydrodynamischen Fall eine Gebietscharakterisierung ermöglichen. Die Idee dieser Modifikationen basiert auf der Tatsache, daß für das Einfachschichtpotential einer Dichte $\psi$ mit $\int_{\partial D} \psi(x) d s(x)=0$ gilt

$$
\begin{aligned}
S \psi(x) & =\frac{1}{4 \pi} \int_{\partial D}\left(\ln \frac{1}{|x-y|} \psi(y)+\frac{(x-y) \cdot \psi(y)}{|x-y|^{2}}(x-y)\right) d s(y) \\
& =\frac{1}{4 \pi} \int_{\partial D} \ln \left|\frac{x}{x-y}\right| \psi(y) d s(y)+o(1)=o(1), \quad|x| \rightarrow \infty
\end{aligned}
$$

und somit ist die Greensche Formel im unbeschränkten Gebiet $D^{+}$auf $S \psi$ anwendbar.

\subsubsection{Faktorisierung des Meßoperators}

Wie im dreidimensionalen Fall sei der Operator $G_{2}:\left.f \rightarrow u\right|_{\partial B}$ als Abbildung der $L^{2}$-Randdaten auf die $\partial B$-Einschränkung der Lösung des zweidimensionalen $\ddot{A} \mathrm{DP}$ eingeführt.

Aus 2.34 und 2.36 gewinnt man für $G_{2}$ die Darstellung

$$
G_{2}=U_{2}\left(-\frac{1}{2} I+K_{(\partial D)}+L-\frac{|\partial D|}{2 \pi} M\right)^{-1}
$$

mit dem Operator

$$
\left(U_{2} \varphi\right)(x):=\left.\left(K_{2}+L-\frac{|\partial D|}{2 \pi} M\right)\right|_{\partial B} .
$$

Zusätzlich betrachten wir die folgende Modifizierung des Operators $G_{2}$ : der Operator $\widetilde{G}_{2}: L^{2}(\partial D) \rightarrow L^{2}(\partial B)$ sei gegeben durch

$$
\widetilde{G_{2}} f=G_{2} f-u_{\infty},
$$

d.h. von der auf $\partial B$ gemessenen Lösung wird deren Wert im Unendlichen abgezogen. 
Aus dem Ausdruck 3.16 für $G_{2}$ leiten wir für $\widetilde{G}_{2}$ die folgende Darstellung ab:

$$
\widetilde{G}_{2}=\widetilde{U}_{2}\left(-\frac{1}{2} I+K_{(\partial D)}+L-\frac{|\partial D|}{2 \pi} M\right)^{-1}
$$

mit

$$
\left(\widetilde{U}_{2} \varphi\right)(x):=\left.\left(K_{2}+L\right)\right|_{\partial B}
$$

Für den adjungierten Operator $\widetilde{G}^{*}: L^{2}(\partial B) \rightarrow L^{2}(\partial D)$ gilt entsprechend

$$
\widetilde{G}_{2}^{*}=\left(-\frac{1}{2} I+K_{(\partial D)}^{\prime}+L^{*}-\frac{|\partial D|}{2 \pi} M\right)^{-1} \widetilde{U}_{2}^{*}
$$

Dabei hat der zu $\widetilde{U}$ adjungierter Operator $\widetilde{U}_{2}^{*}$ die Gestalt $\widetilde{U}_{2}^{*}=\widetilde{K^{\prime}}+L_{\partial B}^{*}$, wobei $\widetilde{K^{\prime}}$ für den Operator $K^{\prime}$ mit $\partial D$ ersetzt durch $\partial B$ steht und $L_{\partial B}^{*}: L^{2}(\partial B) \rightarrow L^{2}(\partial D)$ gegeben ist durch

$$
\left(L_{\partial B}^{*} \varphi\right)(x):=\frac{1}{2 \pi}\left(x_{2},-x_{1}\right)^{T} \int_{\partial B} \varphi(y) \cdot \frac{\left(y_{2},-y_{1}\right)^{T}}{|y|^{2}} d s(y), \quad x \in \partial D .
$$

\section{Satz 3.8}

Der lineare Operator $G_{2}: L^{2}(\partial D) \rightarrow L^{2}(\partial B)$ ist beschränkt und injektiv.

Der Nullraum des Operators $\widetilde{G_{2}}$ ist gegeben durch

$$
N\left(\widetilde{G}_{2}\right)=\operatorname{span}\left\{e_{1}, e_{2}\right\}
$$

Beweis:

Den Beweis der ersten Aussage führen wir analog zum dreidimensionalen Fall (vgl. Satz 3.1). Unter Berücksichtigung der Injektivität des Operators

$$
\left(-\frac{1}{2} I+K_{(\partial D)}+L-\frac{|\partial D|}{2 \pi} M\right)^{-1}
$$

erhält man aus der Darstellung 3.16 von $G_{2}$, daß $N\left(G_{2}\right)=N\left(U_{2}\right)$ gilt.

Nun erfülle ein Vektorfeld $\varphi \in L^{2}(\partial D)$ die Gleichung $U_{2} \varphi=0$. Für die Funktion

$$
u(x):=\left(K+L-\frac{|\partial D|}{2 \pi} M\right) \varphi, \quad x \in D^{+}
$$

gilt nach Voraussetzung $\left.u\right|_{\partial B}=U_{2} \varphi=0$ und damit wegen der Eindeutigkeit des $\ddot{A ̈ D P}$ auch $u=0$ in $D^{+}$. Die Anwendung der $L^{2}$-Sprungbeziehung für das Doppelschichtpotential führt jetzt zu

$$
\left(-\frac{1}{2} I+K_{(\partial D)}+L-\frac{|\partial D|}{2 \pi} M\right) \varphi=0
$$

was nur für $\varphi=0$ möglich ist. 
Gilt $\widetilde{G}_{2} f=G_{2} f-u_{\infty}=0$ für ein $f \in L^{2}(\partial D)$,so erhält man nach der Eindeutigkeit des $\ddot{A D P}$ und der Linearität von $G_{2}$ die Gleichung $G_{2}\left(f-u_{\infty}\right)=0$, woraus mit der Injektivität von $G_{2}$ die Beziehung $f=u_{\infty}=$ const folgt. Damit ist die zweite Aussage des Satzes bewiesen.

\section{Satz 3.9}

Der Operator $F_{2}: L^{2}(\partial B) \rightarrow L^{2}(\partial B)$ besitzt die Darstellung

$$
F_{2}=\widetilde{G} S_{0} \widetilde{G}^{*}
$$

mit

$$
S_{0}:=(I-M) S(I-M)+M
$$

und ist kompakt. Darüber hinaus ist $F_{2}$ positiv semi-definit. Als Abbildung von $H^{-\frac{1}{2}}(\partial B)$ hat $F_{2}$ den Nullraum

$$
N\left(F_{2}\right)=\operatorname{span}\left\{\nu_{\partial B}, \psi_{1}, \psi_{2}\right\}
$$

mit den durch $\psi_{j}=S_{\partial B}^{-1} e_{j}, j=1,2$ gegebenen Vektorfeldern $\psi_{1}, \psi_{2} \in H^{-\frac{1}{2}}(\partial B)$.

Beweis: Für alle $y \in \partial B$ gilt

$$
-\left.w(\cdot, y)\right|_{\partial B}+w_{\infty}(y)=\left.\widetilde{G} \Psi^{(r, c)}(\cdot-y)\right|_{\partial D}
$$

Dieses, multipliziert mit einem $g \in L^{2}(\partial B)$ und integriert über $\partial B$, liefert wegen der guten Gestelltheit des Äußeren Dirichlet Probems und der Beschränktheit des linearen Operators $\widetilde{G}$

$$
F_{2}=\widetilde{G} A=\widetilde{G}(I-M) A
$$

denn es gilt $\widetilde{G} M=0$. Hierbei ist der Hilfsoperator $A: L^{2}(\partial B) \rightarrow L^{2}(\partial D)$ gegeben durch

$$
(A g)(x):=\int_{\partial B} \Psi^{(r, c)}(x-y) g(y) d s(y), \quad x \in \partial D .
$$

Wegen $M(I-M) \varphi=0$ verschwindet das Einfachschichtpotential mit der Dichte $(I-M) \varphi$ im Unendlichen, es folgt also (mit Berücksichtigung von 3.17)

$$
A^{*}(I-M)=\widetilde{G}_{2} S(I-M)=\widetilde{G}_{2}[(I-M) S(I-M)+M]
$$

und damit

$$
(I-M) A=S_{0} \widetilde{G}_{2}^{*} .
$$


Jetzt überzeugen wir uns, daß der Operator $S_{0}$ in $(3.19)$ positiv definit ist. Definiert man für $\varphi \in C(\partial D)$ das Einfachschichtpotential $(v, p)$ der Dichte $(I-M) \varphi$, so gilt

$$
\begin{aligned}
\left(S_{0} \varphi, \varphi\right) & =\int_{\partial D}((I-M) \varphi \cdot S(I-M) \varphi+\varphi \cdot M \varphi) d s \\
& =\int_{\partial D} v \cdot\left(\left(T_{p}^{v} \nu\right)_{-}-\left(T_{p}^{v} \nu\right)_{+}\right) d s+|\partial D \| M \varphi|^{2} \\
& =2 \int_{\mathbb{R}^{2}} D v: D v d x+|\partial D \| M \varphi|^{2} \geq 0 .
\end{aligned}
$$

Mit dem üblichen Dichtheitsargument folgt $\left(S_{0} \varphi, \varphi\right) \geq 0$ für alle $\varphi \in L^{2}(\partial D)$. Dabei bedeutet $\left(S_{0} \varphi, \varphi\right)=0$, daß die ersten Ableitungen von $v$ im ganzen Raum verschwinden und es gilt $M \varphi=0$, woher wegen der Sprungbeziehungen $\varphi=0$ folgt; also ist $S_{0}$ injektiv.

Aus

$$
\left(F_{2} g, g\right)=\left(S_{0} \widetilde{G}^{*} g, \widetilde{G}^{*} g\right)
$$

folgt jetzt, daß $F_{2}$ positiv semi-definit ist.

Mit einem $g \in N\left(F_{2}\right)$, für das wegen 3.20 und $3.17 A g=$ const auf $\partial D$ gilt, definiere man das Einfachschichtpotential $u$.

Da $\left.u\right|_{\partial D}=$ const $=c_{1} e_{1}+c_{2} e_{2}$ gilt, folgt $u=$ const in $D$ und wegen der Analyzität auch in $\bar{B}$. Somit erhalten wir $S_{\partial B} g=$ const. Wählt man ein $b \in \mathbb{R}$, so daß die Funktion $\tilde{g}=g-\left.c \nu\right|_{\partial B}$ in $L_{\nu}^{2}(\partial B)$ liegt, besagt das Lemma 3.3, daß die Integralgleichung $S \tilde{g}=$ const in $H^{-\frac{1}{2}}(\partial B)$ eindeutig lösbar ist. Für die Dichte $g$ gilt also $g=S^{-1}$ const $+\left.b \nu\right|_{\partial B}=S^{-1}\left(c_{1} e_{1}\right)+S^{-1}\left(c_{2} e_{2}\right)+\left.b \nu\right|_{\partial B}$, d.h.

$$
N\left(F_{2}\right) \in \operatorname{span}\left\{\psi_{1}, \psi_{2},\left.\nu\right|_{\partial B}\right\}
$$

mit $\psi_{i}:=S^{-1}\left(e_{i}\right), i=1,2$.

Die Inklusion $\operatorname{span}\left\{\psi_{1}, \psi_{2},\left.\nu\right|_{\partial B}\right\} \subset N\left(F_{2}\right)$ folgt aus 3.20 .

\section{Satz 3.10}

Es gilt $N\left(\widetilde{G_{2}^{*}}\right)=N\left(F_{2}\right)$.

Beweis:

Aus der Faktorisierung 3.18 des Operators $F$ folgt $N\left(\widetilde{G_{2}^{*}}\right) \subset N\left(F_{2}\right)$. Die umgekehrte Inklusion ergibt sich aus 3.21 unter Berücksichtigung der im Beweis des obigen Satzes gezeigten Injektivität von $S_{0}$. 


\subsubsection{Charakterisierung des Gebiets}

Aus der Kompaktheit und der positiven Semidefinitheit des Operators $F_{2}$ folgt, daß seine orthonormierten Eigenfunktionen

$$
g_{j} \in L^{2}(\partial B), j \in \mathbb{N}
$$

eine vollständige orthonormale Basis im Raum

$$
L_{\diamond}^{2}(\partial B):=\left\{\varphi \in L^{2}(\partial B):\left(\varphi, \nu_{\partial B}\right)_{L^{2}(\partial B)}=\left(\varphi, \psi_{1}\right)_{L^{2}(\partial B)}=\left(\varphi, \psi_{2}\right)_{L^{2}(\partial B)}=0\right\}
$$

darstellen, wobei die entsprechenden Eigenwerte eine Folge von positiven reellen Zahlen

$$
\lambda_{1} \geq \lambda_{2} \geq \ldots>0
$$

bilden.

Mit der gleichen Argumentation wie im Lemma 3.5 folgert man für die Funktionen

$$
\varphi_{n}:=\frac{1}{\sqrt{\lambda_{n}}} S_{0}^{\frac{1}{2}} \widetilde{G}^{*} g_{n}, \quad n=1, \ldots,
$$

daß sie eine vollständige orthonormale Basis von $L_{\diamond}^{2}(\partial D)$ darstellen.

Für den Wertebereich des Operators $\widetilde{G} S_{0}^{\frac{1}{2}}$ gilt ferner:

\section{Lemma 3.11}

Eine Funktion $f \in L_{\diamond}^{2}(\partial B)$ liegt in $\widetilde{G} S_{0}^{\frac{1}{2}}\left(L_{\diamond}^{2}(\partial D)\right)$ dann und nur dann, wenn die Reihe

$$
\sum_{i=1}^{\infty} \frac{\left|\left(f, g_{i}\right)\right|^{2}}{\lambda_{i}}
$$

konvergiert.

Beweis: Man folge den einzelnen Schritten des Beweises von Lemma 3.6.

Jedem Punkt $z \in B$ sei nun das Vektorfeld

$$
f_{z}(x):=\left(\operatorname{grad}\left(\Psi^{(r, c)}(x-z) e_{1}\right)\right) e_{2}, \quad x \in \mathbb{R} \backslash\{z\}
$$

zugeordnet. Diese Felder sind divergenzfrei und genügen der Kompatibilitätsbedingung

$$
\left(f_{z}, \nu\right)_{L^{2}(\partial B)}=0 .
$$

Darüber hinaus verschwinden sie im Unendlichen und sind dadurch zulässige StokesGeschwindigkeitsfelder im Äusseren jeder Umgebung des jeweiligen Punktes z. Betrachtet man den Operator $\widetilde{G}_{\Gamma}$, definiert wie $\widetilde{G}$ aus dem vorhergehenden Abschnitt 
mit $\partial D$ ersetzt durch irgendeine geschlossene Kurve $\Gamma \subset B$, die $z$ in ihrem Inneren enthält, so stellt man $\left.f_{z}(x)\right|_{\partial B} \in \widetilde{G}\left(L^{2}(\Gamma)\right)$ fest. Daher erhält man aus

$$
\widetilde{\widetilde{G}_{\Gamma}\left(L^{2}(\Gamma)\right)}=\left[N\left(\widetilde{G}_{\Gamma}^{*}\right)\right]^{\perp}=L_{\diamond}^{2}(\partial B),
$$

daß gilt $\left.f_{z}(x)\right|_{\partial B} \in L_{\diamond}^{2}(\partial B)$, d.h. $\left.f_{z}(x)\right|_{\partial B}$ sind mit Fourier-Reihen nach den Eigenfunktionen von $F_{2}$ darstellbar.

Um eine dem Satz 3.7 entsprechende Charakterisierung des unbekannten Gebiets $D$ anhand von Spektraldaten von $F_{2}$ herzuleiten, brauchen wir nur noch die folgende Aussage über den Operator $S_{0}$.

Satz 3.12

Der Operator $S_{0}: C^{0, \alpha}(\partial D) \rightarrow C^{1, \alpha}(\partial D)$ ist bijektiv und seine Inverse ist beschränkt.

Beweis: Die Differenz zwischen dem injektiven Operator $S_{0}$ und dem Operator

$$
\widetilde{S_{0}}:=S(I-M)+M
$$

ist kompakt, somit folgt die Aussage des Satzes aus der Riesz-Theorie, wenn wir zeigen, daß $\widetilde{S}_{0}: C^{0, \alpha}(\partial D) \rightarrow C^{1, \alpha}(\partial D)$ bijektiv mit beschränkter Inverser ist. Dies beweisen wir analog zum Satz 7.30 in [Kress1].

Löst eine Funktion $\psi=\left(\begin{array}{c}\psi_{1} \\ \psi_{2}\end{array}\right) \in C^{0, \alpha}(\partial D)$ die Gleichung

$$
\widetilde{S_{0}} \psi=f
$$

mit $f \in C^{1, \alpha}(\partial D)$, so löst sie offensichtlich auch die Gleichung

$$
\frac{d}{d s} \widetilde{S_{0}} \psi+M \widetilde{S_{0}} \psi=\frac{d f}{d s}+M f .
$$

Diese beiden Gleichungen sind sogar äquivalent, denn man kann sich leicht überzeugen, daß der Differentiationsoperator $\frac{d}{d s}+M: C^{1, \alpha}(\partial D) \rightarrow C^{0, \alpha}(\partial D)$ injektiv ist, indem man die Gleichung $\frac{d f}{d s}+M f=0$ integriert.

Mit den bekannten Sprungbeziehungen der ersten Ableitungen des logarithmischen Einfachschichtpotentials (s. Kress1, Theorem 7.28) läßt sich die Differentiation unter dem Integral in $\frac{d}{d s} \widetilde{S_{0}} \psi$ durchführen. Auf diesem Wege erhält man äquivalent zu 3.28 die Gleichung

$$
\begin{aligned}
\left(\begin{array}{cc}
\mathcal{K} & 0 \\
0 & \mathcal{K}
\end{array}\right)\left(\begin{array}{l}
\psi_{1} \\
\psi_{2}
\end{array}\right)+\mathcal{M} \psi-\frac{1}{2} \frac{d}{d s}\left(\begin{array}{cc}
S_{\log } M & 0 \\
0 & S_{\log } M
\end{array}\right)\left(\begin{array}{l}
\psi_{1} \\
\psi_{2}
\end{array}\right) & +M \widetilde{S}_{0} \psi= \\
& =\frac{d f}{d s}+M f
\end{aligned}
$$


Dabei bezeichnet $\mathcal{K}$ den Operator

$$
(\mathcal{K} \varphi)(z):=\frac{1}{4 \pi} \int_{\partial D} \frac{\mathbf{k}(z, \zeta)}{\zeta-z} \varphi(\zeta) d s(\zeta), z \in \partial D
$$

mit dem Kern

$$
\mathbf{k}(z, \zeta)=(\zeta-z) \frac{\partial}{\partial s(z)} \ln \frac{1}{|\zeta-z|},
$$

der Operator $\mathcal{M}$ besitzt die Gestalt

$$
\begin{aligned}
&(\mathcal{M} \psi)(z)= \\
& \frac{1}{4 \pi} \frac{d}{d s}\left(\begin{array}{cc}
\int_{\partial D} d s(\zeta) \frac{\left(\zeta_{1}-z_{1}\right)^{2}}{|\zeta-z|^{2}}(I-M) \cdot & \int_{\partial D} d s(\zeta) \frac{\left(\zeta_{1}-z_{1}\right)\left(\zeta_{2}-z_{2}\right)}{|\zeta-z|^{2}}(I-M) \cdot \\
\int_{\partial D} d s(\zeta) \frac{\left(\zeta_{1}-z_{1}\right)\left(\zeta_{2}-z_{2}\right)}{|\zeta-z|^{2}}(I-M) \cdot & \int_{\partial D} d s(\zeta) \frac{\left(\zeta_{2}-z_{2}\right)^{2}}{|\zeta-z|^{2}}(I-M) \cdot
\end{array}\right)\left(\begin{array}{l}
\psi_{1} \\
\psi_{2}
\end{array}\right)
\end{aligned}
$$

und $S_{\log }$ steht für den logarithmischen Einfachschichtpotentialoperator

$$
\left(S_{\log \varphi} \varphi\right)(z):=\frac{1}{2 \pi} \int_{\partial D} \varphi(\zeta) \ln \frac{1}{|\zeta-z|} d s(\zeta), \quad z \in \partial D
$$

Die Operatoren $\mathcal{M}, \frac{d}{d s} S_{\text {log }} M, M \widetilde{S}_{0}$ sind als Abbildungen $C^{0, \alpha}(\partial D) \rightarrow C^{0, \alpha}(\partial D)$ kompakt: $\frac{d}{d s} S_{\text {log }} M$ und $M \widetilde{S}_{0}$ sind beschränkt und haben endlichdimensionale Wertebereiche, während $\mathcal{M}$ eine Superposition eines beschränkten und eines kompakten Operators darstellt.

Ferner gilt $\mathbf{k} \in C^{0,1,1}(\partial D \times \partial D)$ (s. [Kress1], S.119), also stellt der linken Seite der Gleichung 3.29 entsprechender Operator eine kompakt gestörte Superposition eines Cauchy-Integraloperators und der zweidimensionalen Identitätsmatrix dar. Somit ist die Noethersche Theorie (s. Kress1, Abschnitt 7.4) für die singulären Integraloperatoren erster Art mit Cauchy-Kern auf die Gleichung 3.29 anwendbar. Da der Index einer Operatorgleichung erster Art gleich Null ist, muß nur noch die Injektivität des Operators $\widetilde{S}_{0}$ gezeigt werden, damit auf seine Bijektivität und beschränkte Invertierbarkeit geschlossen werden kann.

Sei $\psi$ eine Lösung der homogenen Form der Gleichung 3.29 und damit eine Lösung von $\widetilde{S_{0}} \psi=0$. Ein mit dieser Dichte gebildetes Einfachschichtpotential

$$
u(x)=\int_{\partial D} \Psi^{(r, c)}(x-y)(I-M) \psi(y) d s(y)+M \psi, \quad x \in \mathbb{R}^{2} \backslash \partial D
$$


löst entsprechend das innere sowie das äußere Dirichlet-Probleme bei Null-Randdaten, woher wegen der eindeutigen Lösbarkeit der beiden Probleme $u=0$ in $\mathbb{R}^{2} \backslash \partial D$ folgt. Aus $u(\infty)=0$ folgt $M \psi=0$, und die Sprungbeziehungen für das Einfachschichtpotential liefern $\psi=0$ auf $\partial D$.

\section{Anmerkung 3.1}

Im letzen Satz haben wir unter anderem gezeigt, daß man die beschränkte Lösung des äußeren Dirichlet Problems mit dem modifizierten Einfachschichtpotentialansatz der Gestalt 3.30 finden kann. Nach unserer Kenntnis wurde auf dieseTatsache in der Literatur bisher nicht explizit hingewiesen.

Jetzt sind wir in der Lage, die Hauptaussage dieses Abschnitts zu beweisen.

\section{Satz 3.13}

Für $z \in B$ sind die beiden folgenden Aussagen äquivalent:

$$
\begin{aligned}
& \text { 1) } z \in D \\
& \text { 2) } \sum_{j=1}^{\infty} \frac{\left|\left(f_{z}, g_{j}\right)\right|^{2}}{\lambda_{j}}<\infty
\end{aligned}
$$

Beweis: Liegt $z$ in $D$, so ist die Funktion $f_{z}$ die Lösung des äußeren Dirichlet Problems zu den Randdaten $\left.f_{z}\right|_{\partial D}$. Da sie im Unendlichen verschwindet, gilt

$$
\left.G_{2} f_{z}\right|_{\partial D}=\left.\widetilde{G_{2}} f_{z}\right|_{\partial D}=\left.f_{z}\right|_{\partial B} .
$$

Der Satz 3.12 garantiert die Existenz einer Funktion $\varphi \in C(\partial D)$ mit $S_{0} \varphi=\left.f_{z}\right|_{\partial D}$, und wir folgern

$$
\left.f_{z}\right|_{\partial B}=\left.\widetilde{G_{2}} f_{z}\right|_{\partial D}=\widetilde{G_{2}} S_{0}^{\frac{1}{2}} S_{0}^{\frac{1}{2}} \varphi
$$

mit dem für den kompakten und positiv-definiten $S_{0}$ wohldefinierten Wurzeloperator $S_{0}^{\frac{1}{2}}: L^{2}(\partial D) \rightarrow L^{2}(\partial D)$. Nach Lemma 3.11 folgt die Konvergenz der Reihe 3.31 .

Umgekehrt: Konvergiert die Reihe 3.31, so ist durch

$$
\varphi:=\sum_{i=1}^{\infty} \frac{1}{\lambda_{i}}\left(f_{z}, g_{i}\right) \varphi_{i}
$$

ein $L_{\diamond}(\partial D)$-Vektorfeld gegeben. Aus (3.23) und 3.25 folgt, daß für $\varphi$ die Beziehung $\widetilde{G_{2}} S_{0}^{\frac{1}{2}} \varphi=\left.f_{z}\right|_{\partial B}$ gilt. Nach Satz 3.8 erhalten wir wegen der Eindeutigkeit des ÄDP für $B^{+}$und der Analyzität seiner Lösung, daß die Funktion $f_{z}$ bis auf eine additive Konstante die Lösung des ÄDP in $D^{+}$bei den Randdaten $S_{0}^{\frac{1}{2}} \varphi$ darstellt. Sie ist also 
in $D^{+}$nicht singulär, folglich liegt $z$ in $\bar{D}$. Den Fall $z \in \partial D$ schließt man aber mit dem gleichen Argument wie im Satz 3.7 aus: wegen der Singularität von $f_{z}$ im Punkt $z$ gilt $\left.f_{z}\right|_{\partial D} \notin L^{2}(\partial D)$, somit insbesondere $\left.f_{z}\right|_{\partial D} \notin S_{0}^{\frac{1}{2}}\left(L^{2}(\partial D)\right)$. Demzufolge erhält man $f_{z} \notin \widetilde{G} S_{0}^{\frac{1}{2}}\left(L^{2}(\partial D)\right)$, was nach Lemma 3.11 im Widerspruch mit der Konvergenz der Reihe 3.31 steht. 


\section{Kapitel 4}

\section{Numerische Umsetzung}

In diesem Abschnitt soll die praktische Anwendbarkeit der im letzten Kapitel entwickelten Theorie anhand numerischer Beispiele belegt werden.

Nach Satz 3.13 bietet sich für die approximative Lösung des inversen Problems sofort folgender Algorithmus an:

(1) Löse das Eigenwertproblem für $F_{2}$ anhand der Meßdaten.

(2) Untersuche die Konvergenz der Reihe 3.31 für alle Punkte eines ausreichend feinen Gitters in $B$

(3) Nehme alle Punkte, in welchen die Konvergenz vorliegt, in das Bildergebnis auf.

Für unsere Beispiele verwendete Meßdaten haben die folgende Struktur: zu $M$ äquidistanten, auf ganz $\partial B$ verteilten Punkten $z_{i}, i=1, \ldots, M$ lösen wir, wie im 3 . Kapitel beschrieben, die ÄDP mit den Randdaten $-\Psi_{2}^{(r, c)}\left(\cdot, z_{j}\right) \cdot e_{k} k=1,2$. Von den erhaltenen Lösungen $u^{(k)}\left(\cdot, z_{i}\right)$ wird deren Wert im Punkt $\left(10^{14}, 10^{14}\right)$ abgezogen. Die Komponenten von $\tilde{u}_{j i}^{(k)}:=u^{(k)}\left(z_{j}, z_{i}\right)-u^{(k)}\left(\left(10^{14}, 10^{14}\right)^{T}, z_{i}\right)$ werden zusammengefasst zur $2 M \times 2 M$-Matrix

$$
W=\left(\begin{array}{cccccc}
\tilde{u}_{11,1}^{(1)} & \ldots & \tilde{u}_{1 M, 1}^{(1)} & \tilde{u}_{11,2}^{(1)} & \ldots & \tilde{u}_{1 M, 2}^{(1)} \\
\vdots & & \vdots & \vdots & & \vdots \\
\tilde{u}_{M 1,1}^{(1)} & \ldots & \tilde{u}_{M M, 1}^{(1)} & \tilde{u}_{M 1,2}^{(1)} & \ldots & \tilde{u}_{M M, 2}^{(1)} \\
& & & & & \\
\tilde{u}_{M 1,1}^{(2)} & \ldots & \tilde{u}_{1 M, 1}^{(2)} & \tilde{u}_{M 1,2}^{(2)} & \ldots & \tilde{u}_{1 M, 2}^{(2)} \\
\vdots & & \vdots & \vdots & & \vdots \\
\tilde{u}_{M M, 1}^{(2)} & \ldots & \tilde{u}_{M M, 1}^{(2)} & \tilde{u}_{M M, 2}^{(2)} & \ldots & \tilde{u}_{M M, 2}^{(2)}
\end{array}\right)
$$

Diese diskreten Meßdataten erlauben natürlich nur eine approximative Berechnung 
von den Spektraldaten von $F_{2}$. Ist $z(t)$ eine reguläre Parametrisierung von $\partial B$, so ersetzen wir die Aufgabe der Ermittlung der Paare $\left(g_{n}, \lambda_{n}\right), n \in \mathbb{N}$ mit

$$
\begin{aligned}
\left(F_{2} g_{n}\right)(z(t)) & =-\int_{0}^{2 \pi}\left[w(z(t), z(\tau))-w_{\infty}(z(\tau))\right] g_{n}(z(\tau))\left|z^{\prime}(\tau)\right| d \tau \\
& =\lambda_{n} g_{n}(z(t)), t \in(0,2 \pi]
\end{aligned}
$$

durch das Lösen der Eigenwertaufgabe

$$
-\frac{2 \pi}{M} \sum_{j=1}^{M}\left[w\left(z_{i}, z_{j}\right)-w_{\infty}\left(z_{j}\right)\right] \tilde{g_{n}}\left(z_{j}\right)\left|z^{\prime}\left(t_{j}\right)\right|=\tilde{\lambda_{n}} \tilde{g_{n}}\left(z_{i}\right), i, n=1, \ldots, M .
$$

Durch die Umformung von $4.2 \mathrm{zu}$

$$
\begin{array}{r}
-\frac{2 \pi}{M} \sum_{j=1}^{M}\left[w\left(z_{i}, z_{j}\right)-w_{\infty}\left(z_{j}\right)\right] \sqrt{\left|z^{\prime}\left(t_{i}\right)\right|\left|z^{\prime}\left(t_{j}\right)\right|} \tilde{g_{n}}\left(z_{j}\right) \sqrt{\left|z^{\prime}\left(t_{j}\right)\right|} \\
=\tilde{\lambda_{n}} \tilde{g_{n}}\left(z_{i}\right) \sqrt{\left|z^{\prime}\left(t_{i}\right)\right|}, i, n=1, \ldots, M
\end{array}
$$

erhält man das einfach zu lösende (z. B. mit dem QR-Verfahren mit Shifts) Eigenwertproblem zur symmetrischen Matrix mit den Einträgen

$$
\widetilde{W}_{i j}:=\frac{2 \pi}{M} W_{i j} \sqrt{\left|z^{\prime}\left(t_{i}\right)\right|\left|z^{\prime}\left(t_{j}\right)\right|}, \quad i, j=1, \ldots, 2 M
$$

(man berücksichtige $t_{M+i}=t_{i}$ und $\left.\left|z^{\prime}\left(t_{i}\right)\right|>0, \mathrm{i}=1, \ldots, \mathrm{M}\right)$.

Die Konvergenz der Eigenwerte $\alpha_{n}$ der Matrix $(\widetilde{W})_{i, j=1, \ldots, 2 M}$ gegen die Eigenwerte des Operators $F_{2}$ bei $M \rightarrow \infty$ ist durch die Theorie der kollektiv kompakten Operatoren gesichert (s. [ANS], S. 65-66).

\subsection{Rekonstruktion durch Reihenwertvergleich}

Der zweite Algorithmusschritt erfordert es, numerisch zu entscheiden, ob die Reihe 3.31 konvergiert. Dafür stehen uns die endlichen Summen

$$
R_{N}(\zeta):=\sum_{n=1}^{N} \frac{1}{\widetilde{\lambda_{n}}}\left|\frac{2 \pi}{M} \sum_{j=1}^{M} f_{\zeta}\left(z\left(t_{j}\right)\right) \cdot \widetilde{g_{n}}\left(z\left(t_{j}\right)\right)\right| z^{\prime}\left(t_{j}\right)||^{2}, N=1, \ldots, M
$$

zur Verfügung, welche sich nach der Anwendung der Trapezregel für die Auswertung des Skalarprodukts in 3.31 ergeben. Die Stetigkeitserwägungen legen es nahe, die Entscheidung über die Konvergenz von 3.31 für jeden Punkt $\zeta \in B$ aus dem Vergleich des Wertes der Summe $R_{N}(\zeta)$ mit deren Werten in allen anderen Punkten 


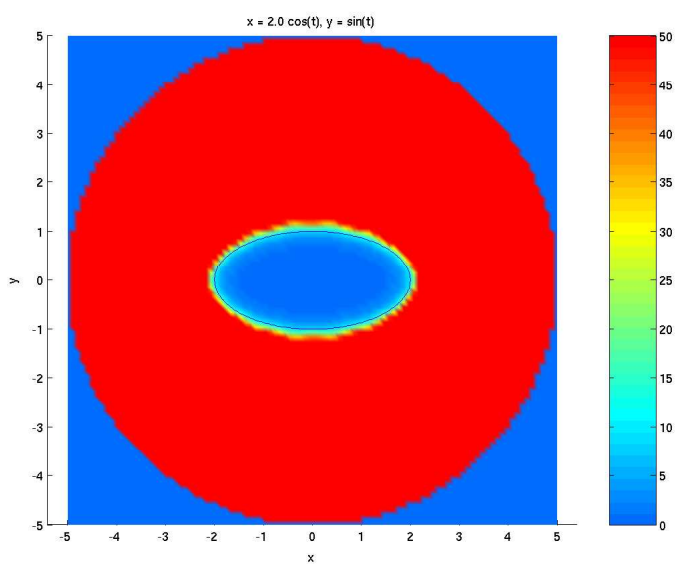

Rekonstruktion einer Ellipse. 64 Meßstellen, $N=40$. Um die Visualisierung zu ermöglichen, mußten wir eine globale obere Schranke für die Werte der Reihe setzen: hier wurden alle Werte über 50 auf 50 gesetzt.

Abbildung 4.1: Ellipse, $\mathrm{N}=40$

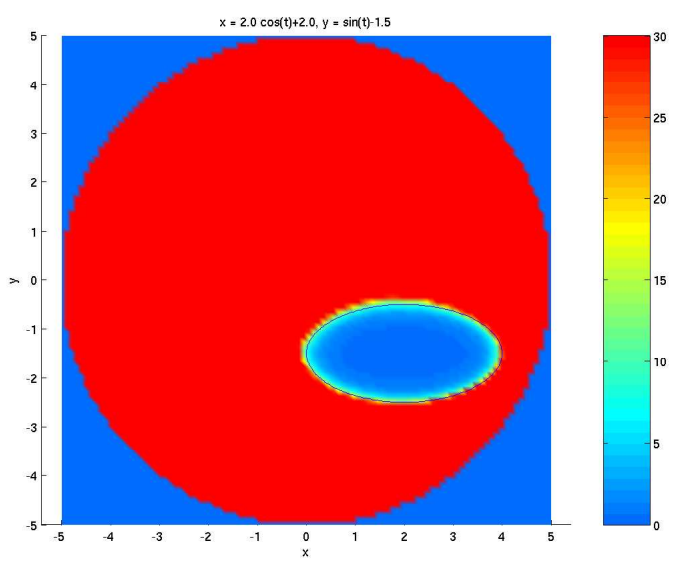

Eine dezentrierte Ellipse. 64 Meßstellen, $N=50$.

Abbildung 4.2: Dezentrierte Ellipse, $\mathrm{N}=50$

zu ziehen: diese sollen innerhalb des zu rekonstruierenden Gebiets vergleichsweise „klein“, außerhalb des Gebiets vergleichsweise „groß“ sein. Nach der intuitiven Wahl einer oberen Wertschranke $S>0$ können dann die Gitterpunkte $z_{i}$ mit $R_{N}\left(\zeta_{i}\right)<S$ dem gesuchten Gebiet zugeordnet werden.

Alternativ können diese Verhältnisse durch die Farbkodierung der Werte der endlichen Reihe in den Auswertungspunkten visualisiert werden. Offensichtlich bedeuten weder die relativ kleinen Werte des endlichen Reihenabschnitts automatisch die Konvergenz der Reihe, noch die großen Werte ihre Divergenz. Brauchbare Ergebnisse lassen sich trotzdem erzielen, wie die Abbildungen 4.1,4.3 zeigen. Als Meßkurve für alle numerischen Tests in diesem Kapitel wurde der Kreis mit Radius 5 genommen.

Die Zahl $N=40$ wurde im nächsten Beispiel aus folgendem Grund genommen: sind $\partial D$ und $\partial B$ analytisch, so fallen die Eigenwerte des Integraloperators $F_{2}$ mindestens 


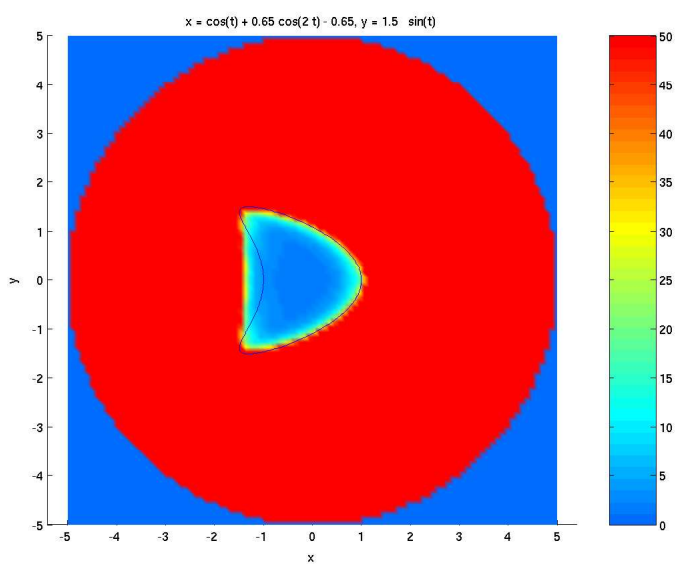

Die Rekonstruktion der konkaven Seite des durch die dünne Linie angedeuteten Objektes („Luftdrachens“) ist nicht optimal. $N=40.200$ Meßstellen.

Abbildung 4.3: Luftdrachen, $\mathrm{N}=40$

exponentiell ab, da sein Kern analytisch ist (s. Satz 15.20 in Kress1]). Für das letzte Beispiel wurde folgende Approximation der Eigenwerte von $F_{2}$ erhalten (hier zum Logarithmus genommen):

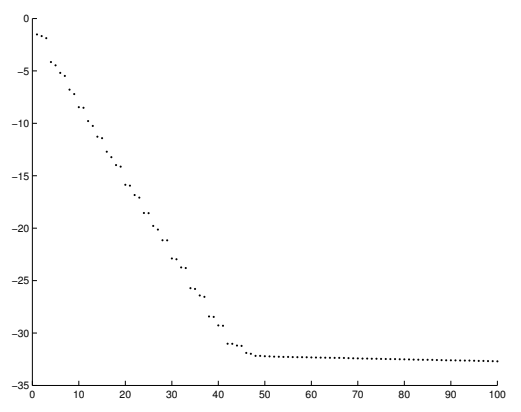

Man sieht, daß sich nur die ersten 40 Eigenwerte gemäß des erwarteten exponentiellen Abklingens verhalten; die restlichen Werte sind unzuverlässig, wohl wegen des Rundungsfehlers.

Abbildung 4.4: Luftdrachen, Eigenwerte

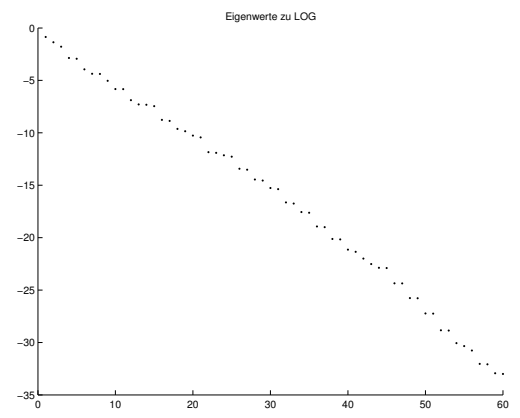

Die Eigenwerte für die dezentrierte Ellipse (s. Abb. 4.1) fallen weniger schnell ab als beim Drachen. Wir können daher mehr Eigenwerte für die Rekonstruktion heranziehen.

Abbildung 4.5: Dezentrierte Ellipse, Eigenwerte 


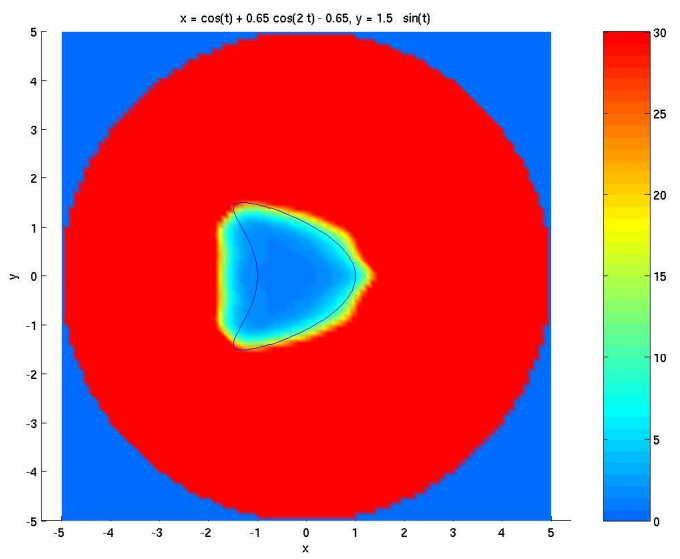

Nimmt man weniger Eigenwerte für die Summenbildung, wird die Rekonstruktion gröber: der Unterschied zwischen den „großen“ und den „kleinen“ Werten bildet sich nicht ausreichend deutlich aus (für dieses Bild wurde $N=20$ gewählt).

Im letzten Beispiel zeigt sich ein leichter Nachteil der benutzten Methode: man kann a priori nicht wissen, wie klein die Reihenwerte sein müssen, um die Reihe als konvergent anzunehmen. Dies erschwert z. B. eine automatisierte Bilderkennung.

\subsection{Das exponentielle Abklingen der Eigenwerte}

Jetzt wollen wir anhand von Beispielen einen anderen Zugang zur numerischen Entscheidung über die Konvergenz der Reihe 3.31 demonstrieren. Dabei benutzen wir [BRH] zufolge das erwähnte exponentielle Abklingen der Eigenwerte, indem wir die Abklingraten der Zähler und der Nenner der Reihe vergleichen. Ist diese Rate für den Zähler größer, als beim Nenner, liegt offenbar eine konvergente Reihe vor.

Dieser Vergleich läßt sich durchführen, indem man die Steigungen der Regressionsgeraden zu den logarithmierten Eigenwerten bzw. Fourierkoeffizienten betrachtet. Diese Vorgehensweise öffnet eine Möglichkeit, die zu erwartende Qualität der Rekonstruktion statistisch zu charakterisieren: anhand der quantitativ bekannten Empfindlichkeit der linearen Regression gegenüber Datenstörungen soll es möglich sein, die Zugehörigkeit der Punkte zum unbekannten Objekt als Hypothese zu überprüfen. Auf jedem Fall hängt der Grad der schlechten Gestelltheit des inversen Problems direkt mit dem exponentiellen Abklingverhalten der Eigenwerte des Operator $F_{2}$ zusammen; das Verwenden der linearen Regression verleiht dem Verfahren gewisse Stabilität.

Das aus den Abbildungen 4.4 und 4.5 ersichtliche Verhalten der Eigenwerte läßt vermuten, daß ihr Auftreten paarweise bei allen zur Ellipse homeomorphen Geometrien des gesuchten Objekts zu erwarten ist. Vor allem im Falle der mit einem zufälligen Fehler gestörten Meßdaten kann man diese Tatsache zu einer weiteren Stabilisierung des Verfahrens gut nutzen, indem man die Werte durch die arithmetische Mittelung paarweise zusammenfasst und erst für diese Daten die Ausgleichsgerade konstruiert. 
Für alle Beispiele dieses Abschnitts wurde auf dem äußeren Quadrat des Kreises mit Radius 5 (die Meßkurve) ein quadratisches $100 \times 100$-Gitter gelegt. Die Auswertung der Reihe erfolgt in dessen Knoten.
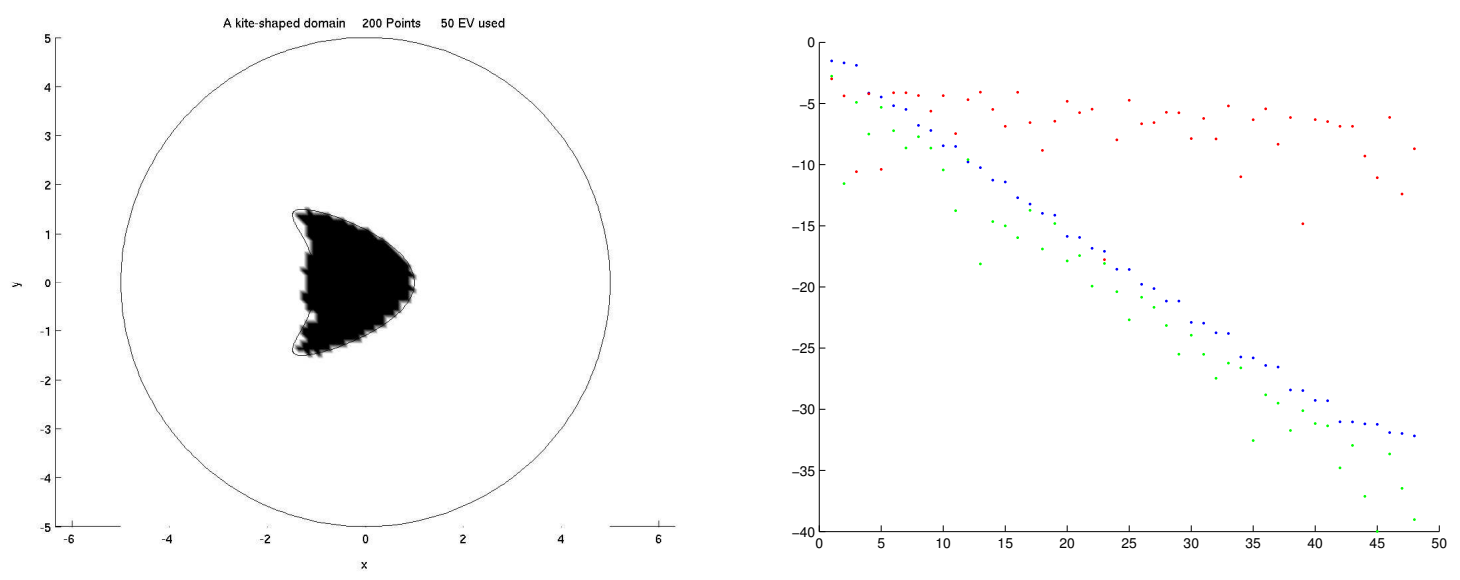

Die linke Abbildung zeigt, welche Qualität der Rekonstruktion auf beschriebenem Wege möglich ist; auch die konkave Seite des Objektes ist korrekt erkennbar. Die Abbildung rechts veranschaulicht das Verhalten der Eigenwerte (blau markiert) und der Zähler der Reihe 3.31 in den Punkten $(0.5,0.5)$ (grün) und $(3,3)$ (rot).

Das folgende Beispiel illustriert die hohe graphische Auflösung des Algorithmus:
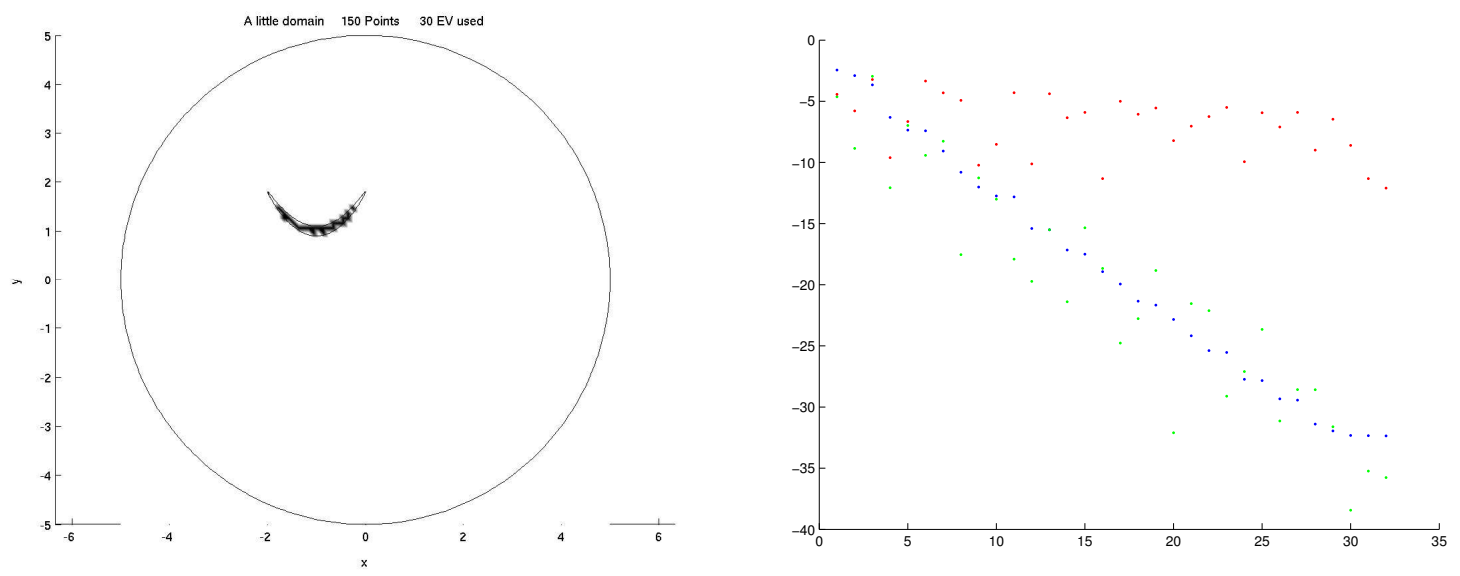

Die rechte Abbildung zeigt das Verhalten der Eigenwerte (blau markiert) und der Zähler der Reihe 3.31 in den Punkten $(-1,1.2)$ (grün) und $(3,3)$ (rot). 


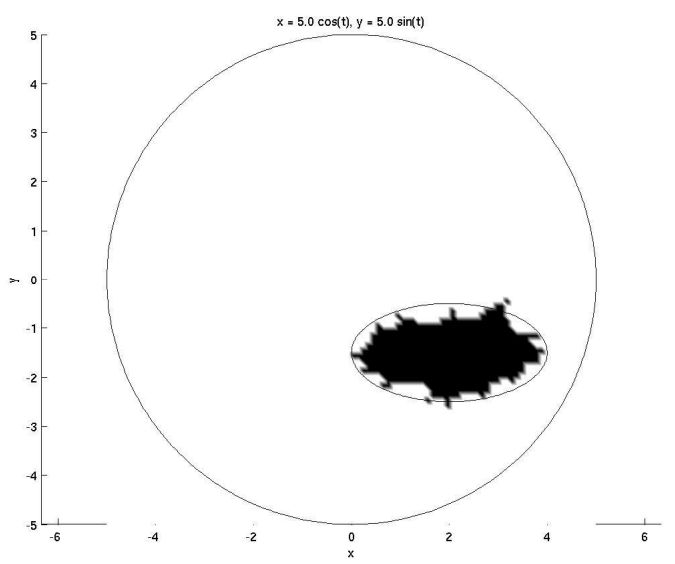

Rekonstruktion einer dezentrierten Ellipse aus gestörten Daten für 64 Meßpunkte. Die Werte der Matrix 4.1 sind um einen standardverteilten Fehler von maximal $0.1 \%$ variiert worden. Natürlich sind bei gestörten Daten weder die Symmetrie der Matrix 4.1 noch ihre positive Definitheit zu erwarten; für dieses Beispiel konnten wir nur die ersten 20 Eigenwerte benutzen, denn die weiteren waren nicht reell.

Abbildung 4.6: Ellipse, 20 Eigenwerte, gestörte Daten

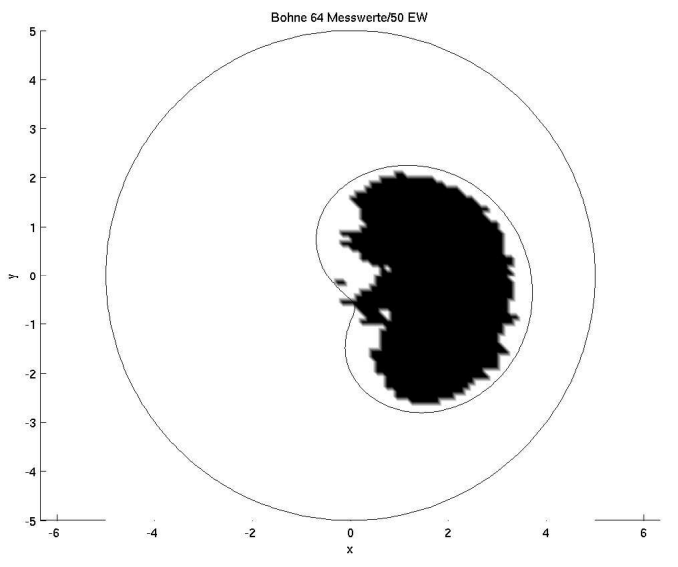

Dieses abschliessende Bespiel macht deutlich, daß die Qualität der Rekonstruktion lokal von der Geometrie des Objektes abhängt. Das Bild links zeigt das Ergebnis der Rekonstruktion von einem asymmetrischen Gebiet (64 Meßpunkte, 50 Eigenwerte) aus ungestörten Daten, die Bilder unten - zwei Rekonstruktionsversuche aus gestörten Daten (24 Eigenwerte, Störung wie im obigen Beispiel).

Abbildung 4.7: Ein asymmetrisches Gebiet
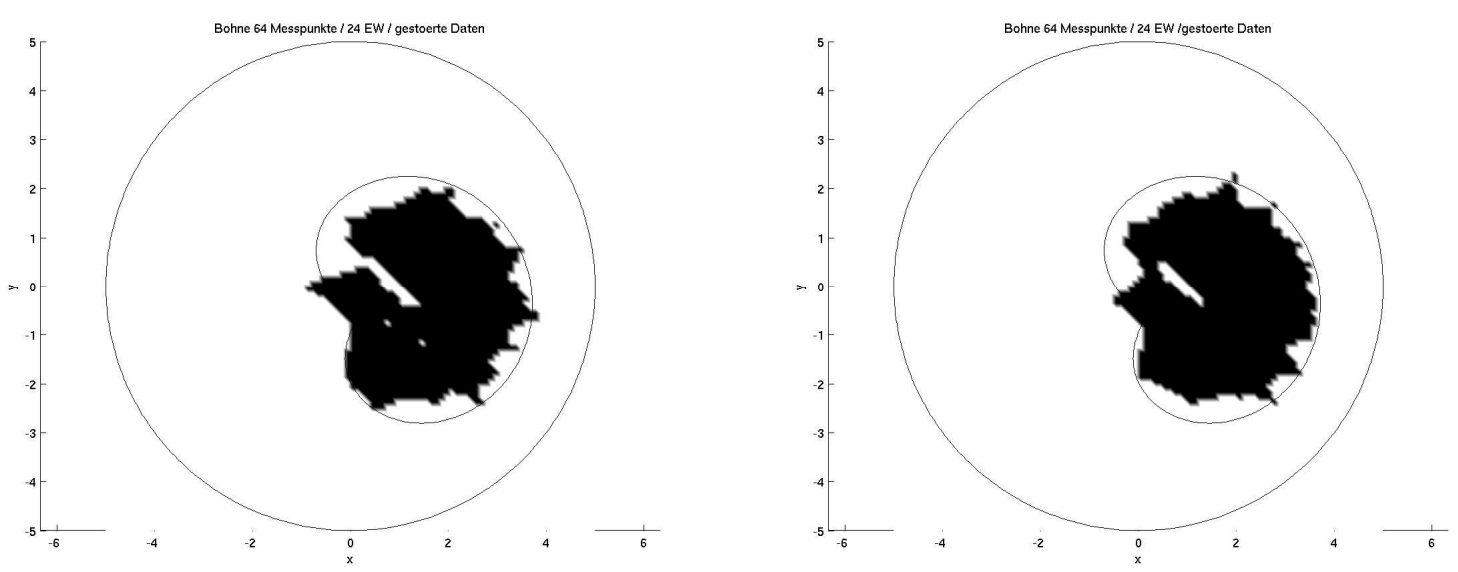

Abbildung 4.8: Ein asymmetrisches Gebiet: Datenstörung 


\section{Literaturverzeichnis}

[AK] C.J.S. Alves, R. Kress: On the far field operator in elastic obstacle scattering. IMA J. Appl. Math. 67, 1-21 (2002)

[ANS] Philip M. Anselone: Collectively Compact Operator Approximation Theory and Applications to Integral Equations. Prentice-Hall, Englewood Cliffs, New Jersey, 1971

[Arens] T. Arens: Linear sampling methods for 2D inverse elastic wave scattering. Inverse Problems 17 1445-1464 (2001)

[Arens/Kirsch] T. Arens, A. Kirsch: The factorization method in inverse scattering from periodic structures. Inverse Problems 19, Number 5, October 2003

[Brühl] M. Brühl: Explicit characterisation of inclusions in electrical impedance tomography. SIAM J. Math. Anal. 32, 1327-1342, 2001

[BRH] M. Brühl, M. Hanke: Numerical implementation of two noniterative methods for locating inclusions by impedance tomography. Inverse Problems 16 (2000) 1029-1042

[Calderón] A. P. Calderón: The Multipole Expansion of Radiation Fields. J. Rat. Mech. Anal. 3, 1954, 523-537

[CCM] D. Colton, J. Coyle, P. Monk: Recent Developments in Inverse Acoustic Scattering Theory. SIAM Review, 42(3): 369-414, 2000

[CHM] D. Colton, H. Haddar, P. Monk: The Linear Sampling Method for Solving the Electromagnetic Inverse Scattering Problem. SIAM Journal on Scientific Computing, 24(3): 719-731, 2002

[Colton/Kress] D. Colton, R.Kress: Integral Equation Methods in Scattering Theory. Wiley, New York, 1983

[Coyle] J. Coyle: Locating the support of objects contained in a two-layered background medium in two dimensions. Inverse Problems, 16(2):275-292, April 2000. 
[Dautray/Lions] R. Dautry, J.-L. Lions: Mathematical Analysis and Numerical Methods for Science and Technology, Vol.4. Springer-Verlag, 1990

[Evans] Evans, J. R.: Randintegralgleichungen bei der Stokes-Gleichung und ihre numerische Behandlung. NAM, Göttingen, Diplomarbeit, 1997

[Fabes] Fabes, C. Kenig and G.Verchota: The Dirichlet problem for the Stokes System on Lipschitz Domains, Duke Math. J. 5 (1988) 769-793.

[Ikehata'98] M. Ikehata: Reconstruction of an Obstacle from the Scattering Amplitude at Fixed Frequency. Inverse Problems 14, 949-954, 1998

[Hähner1] Hähner, P.: An inverse problem in electrostatics. Inverse Problems 14, 1489-1512 (1998)

[Hähner2] Hähner, P.: Eindeutigkeits- und Regularitätssätze für Randwertprobleme bei der skalaren und vektoriellen Helmholtz-Gleichung. NAM, Göttingen, Dissertation 1990

[Hebeker] F.K. Hebeker: Efficient Boundary Element Methods for Threedimensional Exterior Viscous Flow. Num. Meth. Part. Diff. Equations 2, 273$297(1986)$

[HMO] H. Haddar, P. Monk: The linear sampling method for solving the electromagnetic inverse medium problem. Inverse Problems, 18(3): 891-906, Juni 2002

[Hsiao/Kress] G.C. Hsiao, R. Kress: On an integral Equation for the Twodimensional Exterior Stokes Problem. Appl. Num. Math. 1, 77-93 (1985)

[Kersten] Kersten, H.: Grenz- und Sprungrelationen für Potentiale mit quadratsummierbarer Dichte. Resultate d. Math. 3, 17-24 (1980)

[Kirsch1] A. Kirsch: Characterization of the shape of the scattering obstacle by the spectral data of the far field operator. Inverse Problems 14 (1998)

[Kirsch3] A. Kirsch: The MUSIC-Algorithm and the Factorization Method in Inverse Scattering Theory for Inhomogeneous Media. Inverse Problems 18 (2002), 10251040 .

[Lad] O. A. Ladyzhenskaya: The Mathematical Theory of Viscous Incompressible Flow, Second english version. Gordon and Breach 1969

[Kress1] R. Kress: Linear Integral Equations, 2nd ed. Springer-Verlag New-York, 1999

[Kress2] R. Kress: Inverse Boundary Value Problems in Potential Theory. Cubo Matematica Educacional Vol 3. Nr. 2, July 2001 
[Kress3] R. Kress: A Sampling Method for an Inverse Boundary Value Problem for Harmonic Vector Fields. MSRI Preprint No. 2001-029, 2001

[KK] R. Kress, L. Kühn: Linear sampling methods for inverse boundary problems in potential theory. Appl. Numer. Math. 43, 161-173 (2002)

[Luke/Potthast] R. Luke, R. Potthast: The no response test - a sampling method for inverse scattering problems. SIAM Journal of Applied Math, zu erscheinen.

[Mitrea/Taylor] M. Mitrea, M. Taylor: Navier-Stokes Equations on Lipschitz Domains in Riemannian Manifolds. Math. Annalen 321 (2001), 955-987.

[Potthast'01] R. Potthast: Point sources and multipoles in inverse scattering theory. Chapman \& Hall (2001)

[Power/Miranda] H. Power and G. Miranda: 2nd kind integral equation formulation of Stokes flow past a particle of arbitrary shape. SIAM J. App. Math., 47:689698, 1987.

[Power] H. Power: The completed double layer boundary integral equation method for two-dimensional Stokes flow. IMA J. Appl. Math. 51 pp. 123-145 (1993)

[Power/Wrobel] H. Power, L. C. Wrobel: Boundary Integral Methods in Fluid Mechanics. Computational Mechanics Publications Southampton Boston, 1995

[POZ] C. Pozrikidis: Boundary integral and singularity methods for linearized viscous flow. Cambridge University Press 1992

[Varnhorn] W. Varnhorn: The Stokes Equations. Akademie Verlag 1994 


\section{Curriculum vitae}

Persönliche Daten:

Name:

Geburtsdatum:

Geburtsort:

Familienstand:

Staatsangehörigkeit

Eltern:

Schulbildung:

1/1981 - 6/1989

9/1989-6/1991

$6 / 1991$

\section{Studium:}

9/1991-6/1995

$3 / 1996-2 / 2000$

1999

02/2000

seit $3 / 2000$
Viktor Tsiporin

9.5.1974

Sankt-Petersburg, Russische Föderation

ledig

deutsch

Sophie Tsiporin, geb. Farberoff, Chemikerin

Felix Tsiporin, Mathematiker

Allgemeinbildende Schule Nr. 301, St.-Petersburg, Russland

Spezialgymnasium Nr. 30, St.-Petersburg, Russland

Abitur

Studium der Mathematik an der Staatlichen Universität St.-Petersburg.

Studium der Mathematik mit Nebenfach Informatik an der Georg-August-Universität Göttingen

Diplomarbeit: " Über ein Transmissionsproblem bei zeitharmonischen elektromagnetischen Wellen in einem orthotropen Medium"

Diplom in Mathematik

Mitglied im Graduiertenkolleg "Strömungsinstabilitäten und Turbulenz" 